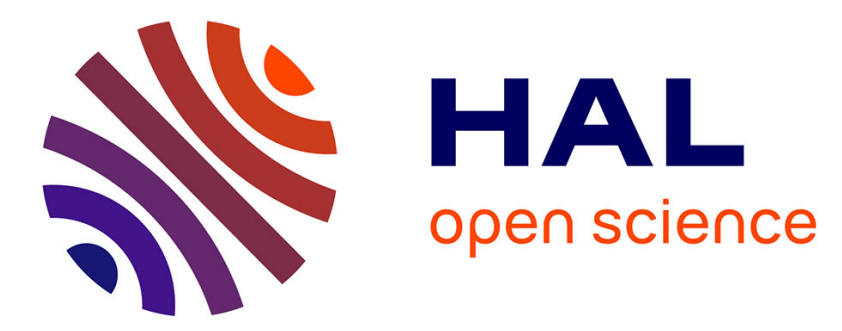

\title{
Market distortions and local indeterminacy: a general approach
}

Teresa Lloyd-Braga, Leonor Modesto, Thomas Seegmuller

\section{To cite this version:}

Teresa Lloyd-Braga, Leonor Modesto, Thomas Seegmuller. Market distortions and local indeterminacy: a general approach. 2011. halshs-00576582

\section{HAL Id: halshs-00576582 \\ https://shs.hal.science/halshs-00576582}

Preprint submitted on 14 Mar 2011

HAL is a multi-disciplinary open access archive for the deposit and dissemination of scientific research documents, whether they are published or not. The documents may come from teaching and research institutions in France or abroad, or from public or private research centers.
L'archive ouverte pluridisciplinaire HAL, est destinée au dépôt et à la diffusion de documents scientifiques de niveau recherche, publiés ou non, émanant des établissements d'enseignement et de recherche français ou étrangers, des laboratoires publics ou privés. 


\section{GREQAM}

Groupement de Recherche en Economie Quantitative d'Aix-Marseille - UMR-CNRS 6579

Ecole des Hautes études en Sciences Sociales Universités d'Aix-Marseille II et III
Document de Travail $n^{\circ}$ 2011-11

\section{Market distortions and local indeterminacy: a general approach}

Teresa Lloyd-Braga

Leonor Modesto

Thomas Seegmuller

March 2011 


\title{
Market distortions and local indeterminacy: a general approach
}

\author{
Teresa Lloyd-Braga ${ }^{1 *}$, Leonor Modesto ${ }^{2}$ and Thomas Seegmuller ${ }^{3}$ \\ ${ }^{1}$ Católica Lisbon - School of Business and Economics \\ ${ }^{2}$ Católica Lisbon - School of Business and Economics and IZA \\ ${ }^{3}$ CNRS and GREQAM
}

March 11, 2011

\begin{abstract}
We provide a methodology to study the role of market distortions on the emergence of indeterminacy and bifurcations. Most of the specific market imperfections considered in the related literature are particular cases of our framework. Comparing them we obtain several equivalence results in terms of local dynamic properties, highlighting the main chanels and classes of distortions responsible for indeterminacy. Our methodolgy consists in introducing general specifications for the elasticities of the crucial functions defining the aggregate equilibrium dynamics of the model. This allows us to study how market distortions influence the range of values for the elasticity of inputs substitution under which local indeterminacy and bifurcations occur. Applying this methodology to the Woodford (1986) framework we find that distortions in the capital market, per se, do not play a major role. We further show that, for empirically plausible values of elasticity of substitution between inputs, indeterminacy requires a minimal degree of distortions. This degree seems to be high under output market distortions, while with labor market distortions the required degree is empirically plausible.
\end{abstract}

JEL classification: C62, E32.

Keywords: Indeterminacy, endogenous fluctuations, market imperfections, externalities, imperfect competition, taxation.

\footnotetext{
${ }^{*}$ Corresponding Author: correspondence should be sent to Teresa Lloyd-Braga, Católica Lisbon, School of Business and Economics, Palma de Cima, 1649-023 Lisboa, Portugal. e-mail: tlb@clsbe.lisboa.ucp.pt.
} 


\section{Introduction}

Several papers have studied the effects of specific market distortions (linked to externalities, imperfectly competitive markets, or government intervention) on local dynamics. ${ }^{1}$ However, a systematic analysis within a general unified framework, able to compare the importance of different distortions for the emergence of indeterminacy and bifurcations, is still missing. In order to fill this gap, we develop a methodology to study and fully characterize the role of market distortions on the occurrence of local indeterminacy and bifurcations, introducing a general framework that accounts for market distortions without determining a priori their specific source.

Market distortions play a role on the local stability properties of the steady state because they modify the elasticities of the crucial functions characterizing the general equilibrium dynamic equations of the model. So our approach consists in generalizing the elasticities obtained when there are no externalities, no government and markets are perfectly competitive, introducing new parameters that represent distortions. We then study how our distortion parameters influence the values of the elasticities of inputs substitution in production (and of labor supply) under which indeterminacy and bifurcations occur. Most of the usual specific market distortions, and different combinations among them, can be recovered as particular specifications of our distortion parameters and, thereby, our results can be used to have an immediate idea of their potential role on the emergence of indeterminacy.

Even if our approach can be applied to any dynamic general equilibrium model, we consider here a dynamic framework based on the Woodford (1986) perfectly competitive one sector model segmented asset economy with heterogenous agents (capitalists and workers). ${ }^{2}$ In accordance with empirical evidence, we assume that inputs are not weak substitutes, a case where indeterminacy and bifurcations would not occur in the absence of distortions, as shown in Grandmont et al. (1998). In contrast, with distortions, local indeterminacy and bifurcations (Hopf, transcritical and flip) may occur in the presence of sufficiently high capital-labor substitution. Our approach allow us to highlight the main channels through which local indeterminacy and bifurcations emerge. One of the main results is that distortions affecting the real interest rate do not play a major role on local dynamics, while with

\footnotetext{
${ }^{1}$ See the references in Section 4.

${ }^{2}$ This model was introduced by Woodford (1986) and later developed by Grandmont et al. (1998) to account for substitution between inputs. This is a suitable framework for our purpose, since several papers have introduced specific market distortions on product and factor markets in this model. These papers provide examples to apply our general methodology (see Section 4).
} 
distortions modifying the real wage and/or consumption and labor supply decisions, indeterminacy may occur even when they are arbitrarily small. However, in this case, indeterminacy requires arbitrarily large elasticities of inputs substitution (and of labor supply). Hence, indeterminacy can only prevail for values of the elasticity of capital-labor substitution around one (those considered empirically plausible) under a minimal degree of distortions.

To illustrate these results we consider examples of specific distortions, on output, capital and labor markets, that can be represented as particular cases of our framework. Our major findings are that: (i) indeterminacy does not occur with capital market distortions (such as capital income taxation); (ii) indeterminacy requires implausible high output market distortions (such as positive productive externalities or countercyclical market power); and (iii) on the contrary, under labor market distortions (such as unemployment benefits with efficiency wages or unions) indeterminacy and bifurcations occur for empirically relevant values of the parameters. Since, as shown in Grandmont et al. (1998), indeterminacy and bifurcations are linked to emergence of endogenous fluctuations, driven by volatile self-fulfilling expectations, our results suggest that labor market imperfections are the most probable cause of endogenous cycles. Hence, our paper fully answers the research question raised in Grandmont et al. (1998) on whether "features such as increasing returns to scale, imperfect competition, and/or sluggish adjustment of wages or prices, alter the dynamics and may or may not improve the range of parameters that give rise to endogenous fluctuations".

We also show that several different specific market distortions have equivalent representations in terms of our distortions parameters, and therefore influence local dynamics in the same way, sharing the same indeterminacy mechanisms. Moreover, some specific market distortions correspond to symmetric representations of particular forms of taxation and, therefore, their effects on indeterminacy can be eliminated by using an appropriate fiscal policy rule. This result is particularly relevant from a policy point of view, since indeterminacy leads to economic instabilility associated with the emergence of expectations driven cycles.

The rest of the paper is organized as follows. In Section 2 we present our general framework. We study the role of our distortion parameters on local dynamics in Section 3, and apply our results to examples with specific market distortions in Section 4. Section 5 provides concluding remarks. Proofs and technical details are provided in the Appendix. 


\section{The Model}

The dynamic model here considered is based on the perfectly competitive Woodford (1986)/Grandmont et al. (1998) framework. In order to ease the presentation we begin with a brief exposition of this model.

\subsection{The Perfectly Competitive Economy}

In each period $t=1,2, \ldots, \infty$, output is produced under a representative technology $A F\left(K_{t-1}, L_{t}\right)$, where $A>0$ is a scaling parameter, $F(K, L)$ is a strictly increasing concave function, homogeneous of degree one in capital, $K>0$, and labor, $L>0$. From profit maximization, the real interest rate $\rho_{t}$ and the real wage $\omega_{t}$ are respectively equal to the marginal productivities of capital and labor, i.e. $\rho_{t}=A F_{K}\left(K_{t-1}, L_{t}\right) \equiv A \rho\left(K_{t-1} / L_{t}\right)$ and $\omega_{t}=$ $A F_{L}\left(K_{t-1}, L_{t}\right) \equiv A \omega\left(K_{t-1} / L_{t}\right)$.

There are two types of infinitely-lived consumers, workers and capitalists. Both can save through two assets, productive capital and money, the latter given by a fixed amount $M$ at the economy level, constant over time. Capitalists are less impatient than workers and do not supply labor, whereas workers face a finance constraint which prevents them from borrowing against their wage earnings. Focusing on equilibria where the finance constraint is binding and capital is the asset with the greatest return, it follows that only workers hold money (they save all their wage income in money), and capitalists hold the entire stock of capital. The behavior of the representative worker can be summarized by the maximization of $U\left(C_{t+1}^{w} / B\right)-V\left(L_{t}\right)$ subject to the budget constraint $P_{t+1} C_{t+1}=w_{t} L_{t}=M_{t}$, where $P_{t}$ is the price of the final good and $w_{t}$ the nominal wage at period $t, C_{t+1}^{w} \geq 0$ the worker's consumption at period $t+1, B>0$ a scaling parameter, $V(L)$ the desutility of labor in $L \in\left[0, L^{*}\right]$, where $L^{*}$ is the worker's time endowment, and $U\left(C^{w} / B\right)$ the utility of consumption. The solution of this problem is given by the intertemporal trade-off between future consumption and leisure:

$$
\omega_{t+1} L_{t+1} / B=\gamma_{t}
$$

where $\gamma_{t} \equiv \gamma\left(L_{t}\right)$ is the usual offer curve with $\varepsilon_{\gamma}(L) \equiv \gamma^{\prime}(L) L / \gamma(L) \geq 1,{ }^{3}$ and $C_{t+1}^{w}=\omega_{t+1} L_{t+1}$ at the monetary equilibrium, where $w_{t} L_{t}=M$ in every period $t$.

\footnotetext{
${ }^{3}$ It is assumed that $V(L)$ is a continuous function for $\left[0, L^{*}\right]$, and $C^{r}$, with $r$ high enough, $V^{\prime}>0, V^{\prime \prime} \geq 0$ for $\left(0, L^{*}\right)$ and $\lim _{L \rightarrow L^{*}} V^{\prime}(L)=+\infty$, with $L^{*}$ possibly infinite. Also, $U\left(C_{t+1}^{w} / B\right)$ is a continuous function of $C_{t+1}^{w} \geq 0$, and $C^{r}$, with $r$ high enough, $U^{\prime}>0, U^{\prime \prime} \leq 0$ for $C_{t+1}^{w}>0$, and $-x U^{\prime \prime}(x) / U^{\prime}(x)<1$. All this implies that $\varepsilon_{\gamma}(L) \geq 1$.
} 
The representative capitalist maximizes the log-linear lifetime utility function $\sum_{t=1}^{\infty} \beta^{t} \ln C_{t}^{c}$ subject to the budget constraint $C_{t}^{c}+K_{t}=(1-\delta+$ $\left.r_{t} / P_{t}\right) K_{t-1}$, where $C_{t}^{c}$ represents his consumption at period $t, \beta \in(0,1)$ his subjective discount factor, $r_{t}$ the nominal interest rate and $\delta \in(0,1)$ the depreciation rate of capital. Solving the capitalist's problem we obtain the capital accumulation equation

$$
K_{t}=\beta\left[1-\delta+\rho_{t}\right] K_{t-1}
$$

A perfectly competitive intertemporal equilibrium is a sequence $\left(K_{t-1}, L_{t}\right)$ $\in R_{++}^{2}, t=1,2, \ldots, \infty$, that, for a given $K_{0}>0$, satisfies (1) and (2), with $\gamma_{t} \equiv \gamma\left(L_{t}\right), \omega_{t} \equiv A \omega\left(K_{t-1} / L_{t}\right)$ and $\rho_{t} \equiv A \rho\left(K_{t-1} / L_{t}\right)$.

Denoting by $\varepsilon_{X, j}$ the elasticity of the function $X$ with respect to $j=K, L$ evaluated at the steady state, the elasticities of the real wage, the real interest rate and the offer curve, with respect to capital and labor, are given by

$$
\begin{aligned}
& \varepsilon_{\rho, K}=-\frac{1-s}{\sigma}, \varepsilon_{\rho, L}=\frac{1-s}{\sigma} \\
& \varepsilon_{\omega, K}=\frac{s}{\sigma}, \varepsilon_{\omega, L}=-\frac{s}{\sigma} \\
& \varepsilon_{\gamma, K}=0, \varepsilon_{\gamma, L}=\varepsilon_{\gamma}
\end{aligned}
$$

where $\varepsilon_{\gamma}-1 \geqslant 0$ represents the inverse of the elasticity of (private) labor supply of the representative worker and, for the representative firm, $s \in(0,1)$ is the elasticity of output with respect to (private) capital and $\sigma>0$ is the elasticity of (private) capital-labor substitution, all evaluated at the steady state. $^{4}$

\subsection{The General Framework}

We now present our general framework with market distortions, explaining and motivating the main differences with respect to the perfectly competitive case. First, in many models characterized by market imperfections, the real interest rate and/or the real wage relevant to the consumers' decisions are no longer equal to the perfectly competitive marginal productivities of capital and labor. This will happen for example in the cases of productive externalities, imperfect competition in the product market or with consumption, labor or capital taxation. Second, with some market imperfections, like in

\footnotetext{
${ }^{4}$ Note that $F\left(K_{t-1}, L_{t}\right)$ can be writen as $L_{t} f\left(K_{t-1} / L_{t}\right)$ so that $s \equiv$ $f^{\prime}(K / L) K / L / f(K / L)$ and $\frac{1}{\sigma} \equiv-\frac{f(K / L) f^{\prime \prime}(K / L) K / L}{f^{\prime}(K / L)\left[f(K / L)-f^{\prime}(K / L) K / L\right]}$. See Grandmont et al. (1998) for more details.
} 
the case of consumption or government spending externalities on preferences, the relevant intertemporal choice of workers becomes a choice between future effective consumption ${ }^{5}$ (that no longer coincides with the wage bill) and leisure. Third, with leisure externalities or in the presence of labour market imperfections, such as unemployment benefits and efficiency wages or unions, the private offer curve derived for the perfectly competitive economy is no longer valid at the social level (see the examples provided in Section 4).

To take these considerations into account, we propose a more general equilibrium dynamic system, given by (4)-(5) in Definition 1 below. Our approach consists in replacing, in the reduced equilibrium dynamic system (1)-(2), the real interest rate $\rho_{t}$, the real wage $\omega_{t}$, and the offer curve $\gamma_{t}$ by three more general functions: $\varrho_{t}$, representing the real interest rate relevant to capitalists' decisions, $\Omega_{t}$ representing effective consumption per unit of labor and $\Gamma_{t}$ representing a generalized offer curve. Since we focus on local dynamics, the elasticities of these three functions play a key role to understand how market distortions affect indeterminacy. We assume that they are defined by expressions which are more general than those obtained under perfect competition (see (3)), introducing a set of new parameters $\left(\alpha_{i j}, \beta_{i j}\right.$, with $i=\{\varrho, \Omega, \Gamma\}$ and $j=\{K, L\})$ that represent a large class of specific market distortions.

Definition 1 A perfect foresight intertemporal equilibrium of our economy, which encompasses market distortions, is a sequence $\left(K_{t-1}, L_{t}\right) \in R_{++}^{2}, t=$ $1,2, \ldots, \infty$, that for a given $K_{0}>0$ satisfies:

$$
\begin{gathered}
K_{t}=\beta\left[1-\delta+\varrho_{t}\right] K_{t-1} \\
(1 / B) \Omega_{t+1} L_{t+1}=\Gamma_{t}
\end{gathered}
$$

where $\varrho_{t} \equiv A \varrho\left(K_{t-1}, L_{t}\right), \Omega_{t} \equiv A \Omega\left(K_{t-1}, L_{t}\right)$ and $\Gamma_{t} \equiv \Gamma\left(K_{t-1}, L_{t}\right)$. The functions $\varrho(K, L), \Omega(K, L)$ and $\Gamma(K, L)$ are positively valued and differentiable as many times as needed for $(K, L) \in R_{++}^{2}$, such that

$$
\begin{aligned}
& \varepsilon_{\varrho, K}=\alpha_{K K}+\frac{\beta_{K K}}{\sigma}-\frac{1-s}{\sigma}, \varepsilon_{\varrho, L}=\alpha_{K L}+\frac{\beta_{K L}}{\sigma}+\frac{1-s}{\sigma} \\
& \varepsilon_{\Omega, K}=\alpha_{L K}+\frac{\beta_{L K}}{\sigma}+\frac{s}{\sigma}, \varepsilon_{\Omega, L}=\alpha_{L L}+\frac{\beta_{L L}}{\sigma}-\frac{s}{\sigma} \\
& \varepsilon_{\Gamma, K}=\alpha_{\Gamma K}+\frac{\beta_{\Gamma K}}{\sigma}, \varepsilon_{\Gamma L}=\alpha_{\Gamma L}+\frac{\beta_{\Gamma L}}{\sigma}+\varepsilon_{\gamma},
\end{aligned}
$$

\footnotetext{
${ }^{5}$ By effective consumption we mean the argument of the utility for consumption, which in the presence of consumption or public spending externalities on preferences will also include them.
} 
where $\alpha_{i, j} \in R$ and $\beta_{i, j} \in R$, for $i=K, L, \Gamma$ and $j=K, L$, are parameters independent of $\varepsilon_{\gamma}$ and $\sigma$.

As under perfect competition, the dynamics of the economy with market distortions are governed by a two dimensional system in capital and labor, where the first equation represents capital accumulation and the second one the intertemporal choice of workers. The perfectly competitive case is recovered from Definition 1 for $\varrho(K, L)=A F_{K}(K, L), \Omega(K, L)=A F_{L}(K, L)$ and $\Gamma(K, L)=\gamma(L)$, and the perfectly competitive elasticities are also recovered from (6) with $\alpha_{i j}=\beta_{i j}=0$ for all $i$ and $j$ (see (3)). Hence, in each equality of (6), the term $\alpha_{i j}+\beta_{i j} / \sigma \neq 0$ represents market distortions, which add two new components to the different elasticities: $\alpha_{i j}$ which provides a measure of the importance of market distortions when inputs are high substitutes in production $\left(\sigma\right.$ high), and $\beta_{i j}$, which become more relevant when inputs are weak substitutes in production ( $\sigma$ low).

\section{$3 \quad$ Local Stability Properties}

\subsection{Non Weak Substitutability of Inputs ( $\sigma$ high)}

Grandmont et al (1998), assuming that $(1-s) \theta<s<1 / 2$ where $\theta \equiv 1-$ $\beta(1-\delta) \in(0,1)$, have shown that local indeterminacy and bifurcations cannot occur under perfect competition if inputs are not weak substitutes, namely if $\sigma>s$. Later on, several works that introduced specific distortions in this framework have shown that, with distortions, indeterminacy may occur with sufficiently substitutable inputs if the private elasticity of labor supply is high enough. ${ }^{6}$ In this paper, considering that inputs are not weak substitutes, we generalize and systematize these previous results, characterizing the effects of market imperfections on the range of values for $\sigma$ and $\varepsilon_{\gamma}$ under which local indeterminacy and bifurcations occur, highlighting several important aspects not yet emphasized or obtained in the related literature.

The assumption that inputs are not weak substitutes is plausible. Empirical studies show that the wage bill is increasing in labor, which, in the absence of market distortions, implies that $\sigma>s$ and means that consumption is increasing in labor. We extend this assumption to our economy with distortions, assuming that effective consumption $(\Omega L)$ is increasing in labor, i.e. $1+\epsilon_{\Omega, L}>0$. From (6), this implies that $\alpha_{L L}>-1$ and $\sigma>\frac{s-\beta_{L L}}{1+\alpha_{L L}}$. Also, since calibrated values for the capital share of output (which, in the

\footnotetext{
${ }^{6}$ See, for instance, Cazzavillan et al. (1998), Barinci and Chéron (2001), Lloyd-Braga and Modesto (2007), and other examples provided in Section 4.
} 
absence of distortions, is represented by $s$ ) are usually lower than $1 / 2$, and since values for $\theta$ are rather small when the period is short, we assume that $1 / 2>s>\theta(1-s)$, as typically done in Woodford economies. Finally, we further extend the latter assumption to our economy with distortions assuming that $\beta_{L L}-\theta\left[\left(s-\beta_{L L}\right) \alpha_{K K}+\beta_{K K}-\alpha_{L L}\left(1-s-\beta_{K K}\right)\right]<s-\theta(1-s)$ and $\left|\beta_{i, j}\right|<s$. Both, given the other assumptions, are satisfied in the absence of distortions, or when they are small enough. ${ }^{7}$ Moreover, these inequalities imply that $\alpha_{K K}>-\frac{1}{\theta}$ and $\sigma>\frac{s-\beta_{L L}}{1+\alpha_{L L}}>\frac{\theta\left(1-s-\beta_{K K}\right)}{1+\theta \alpha_{K K}}$, i.e. $1+\theta \epsilon_{\varrho, K}>0$ (see (6)). Hence, under our assumptions, capital income is increasing with capital, as suggested by empirical works. All these assumptions are summarized below in Assumption 1 and we consider them satisfied in the rest of the paper.

\section{Assumption 1}

1. Small distortions, short period and capital share of output small

a. $\left|\beta_{i, j}\right|<s$, with $i=K, L, \Gamma, j=K, L$

b. $0<\theta(1-s)<s<1 / 2$

$$
\beta_{L L}-\theta\left[\left(s-\beta_{L L}\right) \alpha_{K K}+\beta_{K K}-\alpha_{L L}\left(1-s-\beta_{K K}\right)\right]<s-\theta(1-s)
$$

2. Effective consumption increasing in $L: \sigma \in\left(\frac{s-\beta_{L L}}{1+\alpha_{L L}},+\infty\right), \alpha_{L L}>-1$.

Without distortions, Assumption 1 becomes $\sigma>s>(1-s) \theta$ with $s<1 / 2$, and indeterminacy cannot occur, as referred above. Hence, the occurrence of indeterminacy and bifurcations in our framework is due to the existence of market distortions, mainly because of their effects through $\alpha_{i j}$, which are more relevant than those through $\beta_{i, j}$, when inputs are not weak substitutes in production.

\subsection{Log-linearized System}

In order to obtain a full characterization of the local stability properties of the model, we first log-linearize the system (4)-(5) around the normalized steady state $^{8}$, obtaining:

$$
\left[\begin{array}{c}
\widehat{K}_{t} \\
\widehat{L}_{t+1}
\end{array}\right]=\left[\begin{array}{cc}
\left(1+\theta \varepsilon_{\varrho, K}\right) & \theta \varepsilon_{\varrho, L} \\
\frac{\epsilon_{\Gamma, K}-\varepsilon_{\Omega, K}\left(1+\theta \varepsilon_{\varrho, K}\right)}{1+\varepsilon_{\Omega, L}} & \frac{\varepsilon_{\Gamma, L}-\theta \varepsilon_{\varrho, L} \varepsilon_{\Omega, K}}{1+\varepsilon_{\Omega, L}}
\end{array}\right]\left[\begin{array}{c}
\widehat{K}_{t-1} \\
\widehat{L}_{t}
\end{array}\right] \equiv[J]\left[\begin{array}{c}
\widehat{K}_{t-1} \\
\widehat{L}_{t}
\end{array}\right],
$$

\footnotetext{
${ }^{7}$ Notice that the former can also be written as $\theta\left[\left(1+\alpha_{L L}\right)\left(1-s-\beta_{K K}\right)-\left(s-\beta_{L L}\right) \alpha_{K K}\right]<s-\beta_{L L}$, so that, given $\beta_{L L}<s$, it will be satisfied when $\theta$ is small enough.

${ }^{8}$ We consider the normalized steady state $(K, L)=(1,1)$, whose existence is shown in Proposition 9 (see Appendix 6.1).
} 
where hat-variables denote deviation rates from their steady-state.

The local stability properties of the model, being determined by the eigenvalues of the Jacobian matrix $J$ (or, equivalently, by its trace, $T$, and determinant, $D$, as explained below) depend on the values taken by $\varepsilon_{\varrho, j}, \varepsilon_{\Omega, j}$, and $\varepsilon_{\Gamma, j}$. Distortions influence the local dynamics of the model by modifying these elasticities relatively to the perfectly competitive case, through the parameters $\alpha_{i j}$ and $\beta_{i j}$. By direct inspection of (7), we may immediately deduce that distortions affecting the $\varrho$ function do not play a major role, since $\varepsilon_{\varrho, i}$ appears in $J$ always multiplied by $\theta$, a parameter that takes rather small values when the period of time considered is short. In contrast, distortions affecting the $\Gamma$ and/or the $\Omega$ function can significantly influence the dynamic behavior of our system. Major differences with respect to the perfectly competitive case are that, with distortions (see (3) and (6)), $\varepsilon_{\Gamma, L}$ may become lower than $1\left(\alpha_{\Gamma, L}<0\right.$ under $\sigma$ large) and $1+\varepsilon_{\Omega, L}$ may take values greater than 1 ( $\alpha_{L, L}>0$ under $\sigma$ large). As we shall see, due to this, and in contrast to the perfectly competitive case, indeterminacy is possible even with arbitrarily small distortions in the $\Gamma$ and/or the $\Omega$ functions when capital and labor are not weak substitutes $(\sigma$ large $) .{ }^{9}$

We characterize the role of market distortions on local stability properties using the geometrical method developed in Grandmont et al. (1998), i.e. we analyze how $T$ and $D$ change in the space $(T, D)$, according to the different values taken by the parameters of the model. See figures 1 to 3 . Note that $T$ and $D$ correspond, respectively, to the sum and product of the two eigenvalues of $J$, i.e. the two roots of the associated characteristic polynomial $P(\lambda) \equiv \lambda^{2}-\lambda T+D=0$. Hence, along the line $(A B)$, one eigenvalue is equal to -1 , i.e. $P(-1) \equiv 1+T+D=0$. Also, on the line $(A C)$ one eigenvalue is equal to 1 , i.e. $P(1) \equiv 1-T+D=0$, and on the segment $[B C]$, the two eigenvalues are complex conjugates with a unit modulus, i.e. $D=1$ and $|T|<2$. It can be deduced that the steady state is a sink, with both absolute eigenvalues lower than 1 , when $D<1$ and $|T|<1+D$, i.e., when $(T, D)$ is inside the triangle $A B C$. It is a saddle-point, when $|1+D|<$ $|T|$. Otherwise, it is a source (locally unstable). Since $K$ is a predetermined variable (see Definition 1), the steady state is locally indeterminate when it is a sink. These figures are also useful to study the occurrence of bifurcations. Considering that a parameter of the model is made to continuously vary in its admissible range (for instance $\varepsilon_{\gamma} \in[1,+\infty)$ ), a transcritical bifurcation

\footnotetext{
${ }^{9}$ To see this, take as a first approximation $\theta$ arbitrarily close to zero, so that $D \approx$ $\varepsilon_{\Gamma, L} /\left(1+\varepsilon_{\Omega, L}\right)$. Distortions on $\Omega$ and $\Gamma$ easily allow $D$ to take values lower than 1 , a required condition for indeterminacy. On the contrary, under perfect competition this is not possible for $\sigma$ large since from (3) $\varepsilon_{\Gamma, L}=\varepsilon_{\gamma}>1$ and $0<1+\varepsilon_{\Omega, L}=1+\varepsilon_{\omega, L}<1$ for $\sigma>s>\theta(1-s)$.
} 
generically occurs when $(T, D)$ crosses the line $(A C)\left(\varepsilon_{\gamma}\right.$ crossing the critical value $\left.\varepsilon_{\gamma_{T}}\right) .{ }^{10}$ When $(T, D)$ crosses the line $(A B)\left(\varepsilon_{\gamma}\right.$ crossing the critical value $\left.\varepsilon_{\gamma_{F}}\right)$, a flip bifurcation generically occurs. When $(T, D)$ crosses the segment $[B C]$ in its interior $\left(\varepsilon_{\gamma}\right.$ crossing the critical value $\left.\varepsilon_{\gamma_{H}}\right)$, a Hopf bifurcation generically occurs. ${ }^{11}$

In our case the analysis of the changes in $T$ and $D$ is quite complex, since we have 16 parameters and the expressions of $T$ and $D$ are nonlinear in some of them. Therefore, to simplify our task, we impose some conditions on the parameters that are verified, not only under perfect competition, but also for most of the distortions considered in the literature (see Section 4). They are formalized in the following assumptions:

\section{Assumption 2}

$$
\left(\beta_{L K}+s\right)=\frac{\left(1-s-\beta_{K K}\right)\left(s-\beta_{L L}\right)}{\left(1-s+\beta_{K L}\right)} \text { and } \beta_{\Gamma K}=-\beta_{\Gamma L} \frac{1-s-\beta_{K K}}{1-s+\beta_{K L}} .
$$

\section{Assumption 3}

$$
\alpha_{K, K}\left(\alpha_{\Gamma, L}-\alpha_{L, L}\right)=\alpha_{K, L}\left(\alpha_{\Gamma, K}-\alpha_{L, K}\right) \text {, i.e., } 1+D_{1}(+\infty)-T_{1}(+\infty)=0 \text {. }
$$

where $T_{1}$ and $D_{1}$ denote respectively the values of $T$ and $D$ when $\varepsilon_{\gamma}=1$, and $T_{1}(+\infty)$ and $D_{1}(+\infty)$ denote the values of $T_{1}$ and $D_{1}$ when $\sigma$ tends to $+\infty .{ }^{12}$ Using (6)-(7) and Assumptions 2 and $3, T$ and $D$ can be written as follows:

$$
\begin{aligned}
& T=\frac{\sigma}{\sigma\left(1+\alpha_{L L}\right)-\left(s-\beta_{L L}\right)}\left(\varepsilon_{\gamma}-1\right)+T_{1} \text { with } \\
& T_{1} \equiv 1+\left\{\sigma\left[\left(1+\theta \alpha_{K K}\right)\left(1+\alpha_{\Gamma L}\right)-\theta \alpha_{\Gamma K} \alpha_{K L}\right]+\beta_{\Gamma L}\right. \\
& -\theta\left[\left(1+\alpha_{L L}\right)\left(1-s-\beta_{K K}\right)+\alpha_{K K}\left(s-\beta_{L L}\right)+\alpha_{L K}\left(1-s+\beta_{K L}\right)\right. \\
& \left.\left.+\alpha_{K L} \frac{\left(1-s-\beta_{K K}\right)\left(s-\beta_{L L}\right)}{1-s+\beta_{K L}}\right]\right\} /\left\{\sigma\left(1+\alpha_{L L}\right)-\left(s-\beta_{L L}\right)\right\}
\end{aligned}
$$

\footnotetext{
${ }^{10}$ The case of a saddle node bifurcation is ruled out, since we apply our analysis to $(K, L)=(1,1)$ whose existence is persistent, under the usual scaling procedure. Also, related works with constant elasticities $\epsilon_{\gamma}$ and $\sigma$ (eg. Cazzavillan et al. (1998) and Kuhry (2001)) found at most two steady states, which rules out pitchfork bifurcations. Hence, for the sake of simplicity we disregard pitchfork bifurcations.

${ }^{11}$ The expressions of $\varepsilon_{\gamma_{T}}, \varepsilon_{\gamma_{F}}$ and $\varepsilon_{\gamma_{H}}$ are given in Appendix 6.3.4.

${ }^{12}$ Assumption 2 implies that the numerator and the denominator of $T$ and $D$ depend linearly on the elasticity of capital-labor substitution $\sigma$, while Assumption 3, by referring to Figures 1-3, implies that the point $\left(T_{1}\left(+\infty, D_{1}(+\infty)\right)\right.$ is on line $(A C)$.
} 


$$
\begin{aligned}
& D=\frac{\sigma\left(1+\theta \alpha_{K K}\right)-\theta\left(1-s-\beta_{K K}\right)}{\sigma\left(1+\alpha_{L L}\right)-\left(s-\beta_{L L}\right)}\left(\varepsilon_{\gamma}-1\right)+D_{1} \text { with } \\
& D_{1} \equiv\left\{\sigma\left[\left(1+\theta \alpha_{K K}\right)\left(1+\alpha_{\Gamma L}\right)-\theta \alpha_{\Gamma K} \alpha_{K L}\right]+\beta_{\Gamma L}\right. \\
& -\theta\left[\left(1-s-\beta_{K K}\right)\left(1+\alpha_{\Gamma L}\right)+\alpha_{\Gamma K}\left(1-s+\beta_{K L}\right)\right. \\
& \left.\left.-\alpha_{K L} \beta_{\Gamma L} \frac{1-s-\beta_{K K}}{1-s+\beta_{K L}}-\alpha_{K K} \beta_{\Gamma L}\right]\right\} /\left\{\sigma\left(1+\alpha_{L L}\right)-\left(s-\beta_{L L}\right)\right\}
\end{aligned}
$$

For future reference note that, under Assumption 1, $D$ is increasing in $\varepsilon_{\gamma}$. Also, it can be easily checked that $D_{1}$ is a decreasing function of $\sigma$ when $\alpha_{\Gamma L}>\alpha_{\Gamma L}^{* *}$, with $\alpha_{\Gamma L}^{* *}$ defined below in Assumption 4. Since the inequality $\alpha_{\Gamma L}>\alpha_{\Gamma L}^{* *}$ is verified in the absence of distortions (i.e., when $\alpha_{i j}=\beta_{i j}=0$ for all $i$ and $j$ ) or when distortions are sufficiently small (i.e., all $\alpha_{i j}$ and $\beta_{i j}$ sufficiently close to zero), being also satisfied if distortions do not influence $\Gamma$ (i.e., when $\alpha_{\Gamma j}=\beta_{\Gamma j}=0$ for all $j$ ), and in most of the examples presented in Section 4, we will impose it:

\section{Assumption 4}

$$
\begin{aligned}
\alpha_{\Gamma L}>\quad \alpha_{\Gamma L}^{* *} \equiv-1-\frac{\left(1+\alpha_{L, L}\right) \beta_{\Gamma, L}-\theta\left(s-\beta_{L, L}\right) \alpha_{\Gamma, K} \alpha_{K, L}}{\left(s-\beta_{L, L}\right)\left(1+\theta \alpha_{K, K}\right)-\theta\left(1+\alpha_{L, L}\right)\left(1-s-\beta_{K, K}\right)} \\
+\frac{\theta\left(1+\alpha_{L, L}\right)\left[\alpha_{\Gamma, K}\left(1-s+\beta_{K, L}\right)-\alpha_{K, L} \beta_{\Gamma, L} \frac{1-s-\beta_{K, K}}{1-s+\beta_{K, L}}-\alpha_{K, K} \beta_{\Gamma, L}\right]}{\left(s-\beta_{L, L}\right)\left(1+\theta \alpha_{K, K}\right)-\theta\left(1+\alpha_{L, L}\right)\left(1-s-\beta_{K, K}\right)}
\end{aligned}
$$

The following Lemma summarizes the previous discussion:

Lemma 1 Under Assumptions 1-3, $D$ is an increasing function of $\varepsilon_{\gamma} \in$ $[1, \infty)$. Also, under Assumptions 1-4, $D_{1}$ is a decreasing function of $\sigma \in$ $\left(\frac{s-\beta_{L L}}{1+\alpha_{L L}}, \infty\right)$. Furthermore, Assumption 4 is verified whenever any of the following conditions is satisfied: (i) either distortions are (arbitrarily) small or (ii) distortions do not affect $\Gamma$.

Before proceeding with the full characterization of the local stability properties, we discuss some necessary conditions for the occurrence of indeterminacy, that will help us understanding the role of distortions on local dynamics.

\subsection{Necessary Conditions for Indeterminacy}

As stated above, local indeterminacy occurs when the point $(T, D)$ lies inside the triangle $A B C$. Therefore the conditions $D<1, D>T-1$ and $D>-T-1$ are necessary for the occurrence of indeterminacy (See Figure 1). Using Assumption 1 and (8)-(9) we can easily see that under perfect competition, 
when capital and labor are not weak substitutes (i.e. for $\sigma>s>\theta(1-s)$ ), the first two conditions are not satisfied. Hence, we have the following result:

Proposition 1 Under Assumption 1, local indeterminacy is not possible in the absence of distortions.

With distortions, even if they are small enough, this result may be reversed. As shown below, this requires that distortions satisfy the following inequalities:

\section{Assumption 5}

$$
\begin{array}{ll}
\text { 1. } \alpha_{\Gamma L}<\alpha_{\Gamma L}^{H} \equiv \frac{\alpha_{L L}+\theta\left(\alpha_{\Gamma K} \alpha_{K L}-\alpha_{K K}\right)}{1+\theta \alpha_{K K}}, & \text { i.e. } D_{1}(+\infty)<1 \\
\text { 2. } \alpha_{\Gamma L}<\alpha_{\Gamma L}^{*} \equiv\left\{\left[\alpha_{K K}+\alpha_{K L} \frac{\left(1-s-\beta_{K K}\right)}{\left(1-s+\beta_{K L}\right)}\right]\left(s-\beta_{L L}+\beta_{\Gamma L}\right)+\left(1-s-\beta_{K K}\right) \alpha_{L L}\right. \\
\left.+\left(\alpha_{L K}-\alpha_{\Gamma K}\right)\left(1-s+\beta_{K L}\right)\right\} /\left\{1-s-\beta_{K K}\right\}, & \text { i.e. } D_{1}>T_{1}-1 .
\end{array}
$$

Let us first show that, under Assumptions 1-4, the indeterminacy condition $D<1$ requires Assumption 5.1. Since, given Lemma 1, $D$ is increasing in $\varepsilon_{\gamma} \geqq 1, D_{1}$ is the lowest possible value of $D$, i.e., $D>D_{1}$, so that $D<1$

requires $D_{1}<1$. Since $D_{1}$ is decreasing in $\sigma>\frac{s-\beta_{L L}}{1+\alpha_{L L}}$, we further obtain $D_{1}>D_{1}(+\infty)$. Therefore, $D$ can only take values below 1 if $D_{1}(+\infty)<1$, i.e., if Assumption 5.1 is satisfied. In addition, the condition $D_{1}<1$, using (9) and Assumption 1, is equivalent to $\sigma>\sigma_{H_{1}}$, with $\sigma_{H_{1}}$ given by (18) in Appendix 6.3.3. Hence, we obtain the following Proposition:

Proposition 2 Under Assumptions 1-4, indeterminacy requires Assumption 5.1 and $\sigma>\sigma_{H_{1}}$ (so that $D$ may become lower than 1).

Using also Lemma 1, we have the following Corollary:

Corollary 1 Under Assumptions 1-3, indeterminacy requires Assumption 5.1 and $\sigma>\sigma_{H_{1}}$ whenever any of the following conditions is satisfied: (i) either distortions are (arbitrarily) small or (ii) distortions do not affect $\Gamma$.

A direct implication of these results is that if distortions are sufficiently weak and do not influence the $\varrho$ function, then $\alpha_{L L}>\alpha_{\Gamma L}$ is a necessary condition for indeterminacy. Hence, indeterminacy cannot occur with $\alpha_{L L}=$ $\alpha_{\Gamma L}=0$, and the existence of distortions through labor are crucial when distortions are small.

Let us now focus on the role of Assumption 5.2 for the indeterminacy condition $D>T-1$. Using (8)-(9) and Assumption 1, note that $D>T-1$ is 
equivalent to $\frac{\sigma \alpha_{K, K}-\left(1-s-\beta_{K, K}\right)}{\sigma\left(1+\alpha_{L, L}\right)-\left(s-\beta_{L, L}\right)}\left(\epsilon_{\gamma}-1\right)+A>0$, with $A \equiv \frac{\left(\alpha_{\Gamma, L}^{*}-\alpha_{\Gamma, L}\right)\left(1-s-\beta_{K, K}\right)}{\sigma\left(1+\alpha_{L, L}\right)-\left(s-\beta_{L, L}\right)}$. Hence, if $\alpha_{K, K}<\alpha_{K, K}^{*} \equiv\left(1-s-\beta_{K, K}\right) / \sigma, D$ can only be higher than $T-1$ if $A>0$ or, equivalently under Assumption 1, if $\alpha_{\Gamma, L}^{*}>\alpha_{\Gamma, L}$. Accordingly we obtain the following Proposition.

Proposition 3 Under Assumptions 1-3, when $\alpha_{K K} \leq \alpha_{K K}^{*} \equiv\left(1-s-\beta_{K, K}\right) / \sigma$, indeterminacy requires Assumption 5.2 (so that $D$ may become higher than $T-1)$.

Under Assumption 1, $\alpha_{K K}^{*}>0$ and, therefore, the condition $\alpha_{K K} \leq \alpha_{K K}^{*}$ is satisfied whenever $\alpha_{K K} \leq 0$, or $\alpha_{K K}>0$ but sufficiently close to zero. We immediately derive the following Corollary:

Corollary 2 Under Assumptions 1-3, indeterminacy requires Assumption 5.2 if any of the following conditions is satisfied: (i) either $\alpha_{K K} \leq 0$ (ii) or distortions are arbitrarily small (iii) or distortions do not influence $\varrho$.

From Corollary 2, when distortions do not influence $\Gamma$ and $\alpha_{K, K} \leq 0$, Assumption 5.2 implies that at least one of the parameters $\left\{\alpha_{L, L}, \alpha_{L K}, \alpha_{K L}\right\}$ is positive. As an implication, indeterminacy is ruled out if $\alpha_{L, L}, \alpha_{L, K}, \alpha_{K, L}$ and $\alpha_{K, K}$ are all negative.

Corollaries 1 and 2 show that Assumption 5 is required for the occurrence of indeterminacy with small distortions, when inputs are sufficiently substitutes, as required by Assumption 1 .

Using all the results above we can already emphasize the relative importance of each type of distortions. If there are only distortions on $\varrho$, then from Corollary 1 indeterminacy requires $\alpha_{K, K}<0$ (Assumption 5.1) and, therefore, from Corollary 2 indeterminacy requires that $\alpha_{K, L}$ is above a positive lower bound, given by $-\alpha_{K, K}\left(1-s+\beta_{K L}\right) /\left(1-s+\beta_{K K}\right)$ (Assumption 5.2). Accordingly we have the following Proposition:

Proposition 4 Under Assumptions 1-3, if there are only arbitrarily small distortions in the @ function, or if distortions only influence either $\alpha_{K, K}$ or $\alpha_{K, L}$, Assumption 5 cannot be verified and indeterminacy cannot occur.

Indeed, as explained in the beginning of this section, the effects on local dynamics of distortions affecting $\varrho$ are minor, so that market imperfections influencing the $\varrho$ function are not expected to play a major role for the occurrence of indeterminacy. In contrast, since both Assumptions 5.1 and 5.2 can be easily satisfied with only arbitrarily small distortions on $\Omega$ (with $\alpha_{L L}>0$ ) and/or on $\Gamma$ (with $\alpha_{\Gamma L}<0$ ), distortions in these functions play an important role in the emergence of indeterminacy and bifurcations, as shown in the next section. 


\subsection{Results on Indeterminacy and Bifurcations}

The full characterization of the local stability properties of the model in terms of the relevant parameters is provided in Proposition 5 and Table 1 below. Our results are derived assuming also that:

Assumption $6 \alpha_{\Gamma L}>\alpha_{\Gamma L}^{L} \equiv-\frac{2+\alpha_{L L}-\theta\left(\alpha_{\Gamma K} \alpha_{K L}-\alpha_{K K}\right)}{1+\theta \alpha_{K K}}$ i.e. $D_{1}(+\infty)>-1$.

This Assumption is always verified if distortions are small enough, only influence $\varrho$ and/or $\Omega$, or only influence $\Gamma$ provided that $\alpha_{\Gamma L}$ does not take large negative values $\left(\alpha_{\Gamma L}>-2\right) .{ }^{13}$ Hence, in order to simplify the exposition and the analysis, in the following we consider that Assumption 6 is satisfied.

As explained in the Appendix, our results are obtained using the geometrical method developed in Grandmont et al. (1998), i.e. we analyzed how $T$ and $D$ evolve in the space $(T, D)$ as $\varepsilon_{\gamma} \in[1, \infty)$, the bifurcation parameter, continuously varies in its admissible range. Note that Assumptions 4-6 imply that we focus on values for $\alpha_{\Gamma L}$ such that $\operatorname{Max}\left\{\alpha_{\Gamma L}^{* *}, \alpha_{\Gamma L}^{L}\right\}<\alpha_{\Gamma L}<$ $\operatorname{Min}\left\{\alpha_{\Gamma L}^{*}, \alpha_{\Gamma L}^{H}\right\} \cdot{ }^{14}$

Proposition 5 Let $(K, L)=(1,1)$ be the normalized steady state of the dynamic system (4)-(5), as stated in Proposition 9. Consider that Assumptions 1-3 are verified, and that $\operatorname{Max}\left\{\alpha_{\Gamma L}^{* *}, \alpha_{\Gamma L}^{L}\right\}<\alpha_{\Gamma L}<\operatorname{Min}\left\{\alpha_{\Gamma L}^{*}, \alpha_{\Gamma L}^{H}\right\}$ so that Assumptions 4-6 are also satisfied. Let $\alpha_{\Gamma L}$ further take admissible values in intervals, that we call configurations, specified by referring to the critical values $\alpha_{\Gamma L}^{b}$ and $\alpha_{\Gamma L}^{c}$, whose expressions are given in Appendix 6.3.1. Let $\sigma \in\left(\frac{s-\beta_{L L}}{1+\alpha_{L L}},+\infty\right)$ take values in intervals specified by referring to the critical values $\sigma_{T}, \sigma_{F}, \sigma_{H_{1}}, \sigma_{H_{2}}$, and $\sigma_{H_{3}}$ defined in Appendix 6.3.3., and let $\varepsilon_{\gamma} \in[1, \infty)$ take values in intervals specified by referring to the critical values $\varepsilon_{\gamma_{H}}, \varepsilon_{\gamma_{F}}$ and $\varepsilon_{\gamma_{T}}$ given in Appendix 6.3.4. Consider further that Assumptions 7 and 8 are satisfied whenever $\alpha_{K K}>0$, and that Assumption 9 is verified when $\alpha_{K K} \leq 0$ and $\alpha_{\Gamma L}<\alpha_{\Gamma L}^{c}{ }^{15}$ Then, the nature of the steady

\footnotetext{
${ }^{13}$ Assumption 6, together with Assumptions 3 and 5.1, implies that $\left(T_{1}(+\infty), D_{1}(+\infty)\right)$ is a point on the line $(A C)$, between $A$ and $C$. See Figures 1-3. The reader may later check that, in all the examples presented, this Assumption is indeed implied by Assumptions 1 and/or 4 .

${ }^{14} \mathrm{~A}$ sufficient condition for non emptiness of this interval is that distortions (other than those represented by $\alpha_{\Gamma L}$ ), are small enough. Indeed, if $\alpha_{K L}=\alpha_{K K}=\alpha_{L L}=$ $\alpha_{L K}=\alpha_{\Gamma K}=0$ and $\beta_{i j}=0$ for $i=K, L, \Gamma$ and $j=K, L$, we have $\operatorname{Max}\left\{\alpha_{\Gamma L}^{* *}, \alpha_{\Gamma L}^{L}\right\}=$ $-1<\operatorname{Min}\left\{\alpha_{\Gamma L}^{*}, \alpha_{\Gamma L}^{H}\right\}=0$. Therefore, by continuity, $\operatorname{Min}\left\{\alpha_{\Gamma L}^{*}, \alpha_{\Gamma L}^{H}\right\}$ is still higher than $\operatorname{Max}\left\{\alpha_{\Gamma L}^{* *}, \alpha_{\Gamma L}^{L}\right\}$ for values of those parameters close to zero.

${ }^{15}$ These Assumptions are used merely as an exposition device. Assumptions 7 and 8, which are presented in Appendix 6.2 Configuration (i), are only relevant in the case of
} 
state, whether a saddle, a sink or a source, depends upon the values of the parameters $\alpha_{\Gamma L}, \alpha_{K K}, \sigma$ and $\varepsilon_{\gamma}$ belonging to the intervals indicated in Table $1 .{ }^{16}$ Also, whenever the critical value $\varepsilon_{\gamma_{H}}$ (resp. $\varepsilon_{\gamma_{F}}$ or $\varepsilon_{\gamma_{T}}$ ) appears in some row of Table 1 a Hopf bifurcation (resp. a flip or transcritical bifurcation) generically occurs as $\varepsilon_{\gamma}$ crosses the corresponding value.

Proof. See Appendix 6.2

We start the discussion of the results with two remarks. First, Proposition 5 does not cover the case of perfect competition, since Assumption 5 can only be verified if there are distortions. Second, distortions affecting only $\varrho$, through either $\alpha_{K, K}$ or $\alpha_{K, L}$, are also not covered by Proposition 5 , since in this situation, according to Proposition 4, Assumption 5 is not satisfied. In any case, as shown in Propositions 1 and 4, indeterminacy would not occur in those situations.

In contrast, from Table 1, indeterminacy and bifurcations may occur in the presence of market distortions, under Assumptions 1-6. Indeterminacy (sink) requires values of the elasticity of substitution between capital and labor $(\sigma)$ and of the elasticity of labor supply $\left(1 /\left(\varepsilon_{\gamma}-1\right)\right)$ above certain lower bounds. Of course, given Proposition 2, the lower bound on $\sigma$ is higher or equal to $\sigma_{H_{1}}$. Also, Hopf and/or transcritical bifurcations are possible in all configurations.

There are however differences across configurations: (a) The lower bound on $\sigma$ required for indeterminacy is equal to $\sigma_{H_{1}}$ in configurations $(i)-(i i)$, while it is identical to $\sigma_{H 3}>\sigma_{H 1}$ in configuration (iii); (b) Flip bifurcations are only possible in configurations (ii) and (iii); However, (c) indeterminacy can only occur through a flip bifurcation in configuration (iii); (d) Finally, while an upper bound on $\varepsilon_{\gamma}$ is always required for indeterminacy, in configuration (iii) a lower bound on $\varepsilon_{\gamma}$ may be needed in some cases: when $\sigma \in\left(\sigma_{H 3}, \sigma_{F}\right)$, indeterminacy only emerges for $\varepsilon_{\gamma}>\varepsilon_{\gamma F}>1$. The latter result is important per se, since it implies that imposing an infinitely elastic labor supply at the individual level $\left(\varepsilon_{\gamma}=1\right)$ may not be appropriate to fully understand the implications of market distortions on local indeterminacy.

product market imperfections discussed in Section 4.2.1, being verified for reasonable values for $\theta$ and for the distortions parameters (see Table 2). Assumption 9 is presented in Appendix 6.2 Configuration (iii). It is only relevant in the case of labor market imperfections discussed in Section 4.2.3, being verified in all the examples.

${ }^{16}$ In Table 1, when $\alpha_{K K}=0$, the lines with a ${ }^{*}$ disappear if $\sigma_{H_{2}} \in\left(\frac{\left(s-\beta_{L L}\right)}{\left(1+\alpha_{L L}\right)},+\infty\right)$ does not exist, the upper limit of $\sigma$ in the preceding line becoming $+\infty$. See Appendix 6.2 and 6.4 . 


\begin{tabular}{|c|c|c|c|c|c|}
\hline \multirow{2}{*}{$\begin{array}{l}\alpha_{\Gamma L}<\operatorname{Min}\left\{\alpha_{\Gamma L}^{*}, \alpha_{\Gamma L}^{H}\right\} \\
\alpha_{\Gamma L}>\operatorname{Max}\left\{\alpha_{\Gamma L}^{* *}, \alpha_{\Gamma L}^{L}\right\}\end{array}$} & \multirow{2}{*}{\multicolumn{2}{|c|}{$\sigma$}} & \multicolumn{3}{|c|}{$\varepsilon_{\gamma}$} \\
\hline & & & Saddle & Sink & Source \\
\hline $\begin{array}{l}\text { Configuration }(i) \\
\alpha_{\Gamma L}>\alpha_{\Gamma L}^{b}\end{array}$ & $\begin{array}{l}\text { 1) } \alpha_{K, K} \leq 0 \\
* \\
\text { 2) } \alpha_{K, K}>0\end{array}$ & $\begin{array}{l}\left(\frac{s-\beta_{L L}}{1+\alpha_{L L}}, \sigma_{H_{1}}\right. \\
\left(\sigma_{H_{1}}, \sigma_{H_{2}}\right) \\
\left(\sigma_{H_{2}}, \infty\right) \\
\left(\frac{s-\beta_{L L}}{1+\alpha_{L L}}, \sigma_{H_{1}}\right. \\
\left(\sigma_{H_{1}}, \sigma_{T}\right) \\
\left(\sigma_{T}, \infty\right)\end{array}$ & $\begin{array}{l}\left(\varepsilon_{\gamma_{T}}, \infty\right) \\
\left(\varepsilon_{\gamma_{T}}, \infty\right) \\
\left(\varepsilon_{\gamma_{T}}, \infty\right) \\
\left(\varepsilon_{\gamma_{T}}, \infty\right) \\
\left(\varepsilon_{\gamma_{T}}, \infty\right) \\
-\end{array}$ & $\begin{array}{l}- \\
{\left[1, \varepsilon_{\gamma_{H}}\right)} \\
{\left[1, \varepsilon_{\gamma_{T}}\right)} \\
- \\
{\left[1, \varepsilon_{\gamma_{H}}\right)} \\
{\left[1, \varepsilon_{\gamma_{H}}\right)}\end{array}$ & $\begin{array}{l}{\left[1, \varepsilon_{\gamma_{T}}\right)} \\
\left(\varepsilon_{\gamma_{H}}, \varepsilon_{\gamma_{T}}\right) \\
- \\
{\left[1, \varepsilon_{\gamma_{T}}\right)} \\
\left(\varepsilon_{\gamma_{H}}, \varepsilon_{\gamma_{T}}\right) \\
\left(\varepsilon_{\gamma_{H}}, \infty\right)\end{array}$ \\
\hline $\begin{array}{l}\text { Configuration (ii) } \\
\alpha_{\Gamma L}^{c}<\alpha_{\Gamma L}<\alpha_{\Gamma L}^{b}\end{array}$ & $\begin{array}{l}\text { 1) } \alpha_{K, K} \leq 0 \\
\text { * } \\
\text { 2) } \alpha_{K, K}>0\end{array}$ & $\begin{array}{l}\left(\frac{s-\beta_{L L}}{1+\alpha_{L L}}, \sigma_{F}\right) \\
{\left[\sigma_{F}, \sigma_{H_{1}}\right]} \\
\left(\sigma_{H_{1}}, \sigma_{H_{2}}\right) \\
\left(\sigma_{H_{2}}, \infty\right) \\
\left(\frac{s-\beta_{L L}}{1+\alpha_{L L}}, \sigma_{F}\right) \\
{\left[\sigma_{F}, \sigma_{H_{1}}\right]} \\
\left(\sigma_{H_{1}}, \sigma_{T}\right) \\
{\left[\sigma_{T}, \infty\right)}\end{array}$ & $\begin{array}{l}{\left[1, \varepsilon_{\gamma_{F}}\right)} \\
\text { and }\left(\varepsilon_{\gamma_{T}}, \infty\right) \\
\left(\varepsilon_{\gamma_{T}}, \infty\right) \\
\left(\varepsilon_{\gamma_{T}}, \infty\right) \\
\left(\varepsilon_{\gamma_{T}}, \infty\right) \\
{\left[1, \varepsilon_{\gamma_{F}}\right)} \\
\text { and }\left(\varepsilon_{\gamma_{T}}, \infty\right) \\
\left(\varepsilon_{\gamma_{T}}, \infty\right) \\
\left(\varepsilon_{\gamma_{T}}, \infty\right) \\
\text { - }\end{array}$ & $\begin{array}{l}- \\
{\left[1, \varepsilon_{\gamma_{H}}\right)} \\
{\left[1, \varepsilon_{\gamma_{T}}\right)} \\
- \\
- \\
{\left[1, \varepsilon_{\gamma_{H}}\right)} \\
{\left[1, \varepsilon_{\gamma_{H}}\right)}\end{array}$ & $\begin{array}{l}\left(\varepsilon_{\gamma_{F}}, \varepsilon_{\gamma_{T}}\right) \\
{\left[1, \varepsilon_{\gamma_{T}}\right)} \\
\left(\varepsilon_{\gamma_{H}}, \varepsilon_{\gamma_{T}}\right) \\
- \\
\left(\varepsilon_{\gamma_{F}}, \varepsilon_{\gamma_{T}}\right) \\
{\left[1, \varepsilon_{\gamma_{T}}\right)} \\
\left(\varepsilon_{\gamma_{H}}, \varepsilon_{\gamma_{T}}\right) \\
\left(\varepsilon_{\gamma_{H}}, \infty\right)\end{array}$ \\
\hline $\begin{array}{l}\text { Configuration (iii) } \\
\alpha_{\Gamma L}<\alpha_{\Gamma L}^{c}\end{array}$ & $\begin{array}{l}\text { 1) } \alpha_{K, K} \leq 0 \\
* \\
\text { 2) } \alpha_{K, K}>0\end{array}$ & $\begin{array}{l}\left(\frac{s-\beta_{L L}}{1+\alpha_{L L}}, \sigma_{H_{3}},\right. \\
\left(\sigma_{H_{3}}, \sigma_{F}\right) \\
{\left[\sigma_{F}, \sigma_{H_{2}}\right)} \\
\left(\sigma_{H_{2}}, \infty\right) \\
\left(\frac{s-\beta_{L L}}{1+\alpha_{L L}}, \sigma_{H_{3}},\right. \\
\left(\sigma_{H_{3}}, \sigma_{F}\right) \\
{\left[\sigma_{F}, \sigma_{T}\right)} \\
{\left[\sigma_{T}, \infty\right)}\end{array}$ & $\begin{array}{l}{\left[1, \varepsilon_{\gamma_{F}}\right)} \\
\text { and }\left(\varepsilon_{\gamma_{T}}, \infty\right) \\
{\left[1, \varepsilon_{\gamma_{F}}\right)} \\
\text { and }\left(\varepsilon_{\gamma_{T}}, \infty\right) \\
\left(\varepsilon_{\gamma_{T}}, \infty\right) \\
\left(\varepsilon_{\gamma_{T}}, \infty\right) \\
{\left[1, \varepsilon_{\gamma_{F}}\right)} \\
\text { and }\left(\varepsilon_{\gamma_{T}}, \infty\right) \\
{\left[1, \varepsilon_{\gamma_{F}}\right)} \\
\text { and }\left(\varepsilon_{\gamma_{T}}, \infty\right) \\
\left(\varepsilon_{\gamma_{T}}, \infty\right)\end{array}$ & $\begin{array}{l}\left(\varepsilon_{\gamma_{F}}, \varepsilon_{\gamma_{t}}\right. \\
{\left[1, \varepsilon_{\gamma_{H}}\right)} \\
{\left[1, \varepsilon_{\gamma_{T}}\right)} \\
- \\
\left(\varepsilon_{\gamma_{F}}, \varepsilon_{\gamma_{t}}\right. \\
{\left[1, \varepsilon_{\gamma_{H}}\right)} \\
{\left[1, \varepsilon_{\gamma_{H}}\right)}\end{array}$ & $\begin{array}{l}\left(\varepsilon_{\gamma_{F}}, \varepsilon_{\gamma_{T}}\right) \\
\left(\varepsilon_{\gamma_{H}}, \varepsilon_{\gamma_{T}}\right) \\
\left(\varepsilon_{\gamma_{H}}, \varepsilon_{\gamma_{T}}\right) \\
- \\
\left(\varepsilon_{\gamma_{F}}, \varepsilon_{\gamma_{T}}\right) \\
\left(\varepsilon_{\gamma_{H}}, \varepsilon_{\gamma_{T}}\right) \\
\left(\varepsilon_{\gamma_{H}}, \varepsilon_{\gamma_{T}}\right) \\
\left(\varepsilon_{\gamma_{H}}, \infty\right)\end{array}$ \\
\hline
\end{tabular}

Table 1: Local stability properties and bifurcations 
We now discuss the role of the different distortions on the results obtained. We start by noting that, under Assumption 1 the critical value $\alpha_{\Gamma L}^{b}$, which separates configurations $(i)$ and $(i i)$, is negative in the absence of distortions (see (13) in Appendix 6.3.1). Hence, if distortions are small enough we will always obtain configuration $(i)$. Note also that, in the absence of distortions on $\Gamma, \alpha_{\Gamma L}^{b}$ becomes equal to -1 for $\theta \approx 0$. Hence, since the values taken by $\theta$ are typically rather low, configuration $(i)$ is also the relevant one when there are only distortions on $\varrho$, or on $\Omega$, or on both. This is summarized in the following Corollary.

Corollary 3 Assume that Proposition 5 applies. Configuration (i) is always obtained if: i) either distortions are arbitrarily small, or ii) in the absence of distortions on $\Gamma$ for a sufficiently low $\theta$.

As already referred, distortions influencing the $\varrho$ function do not critically influence local dynamics, a result that is also apparent in Proposition 5. Indeed, within each configuration, $\alpha_{K, K}$ being positive or negative does not change the critical lower bound on $\sigma$ above which indeterminacy may occur $\left(\sigma_{H_{1}}\right.$ in Configurations $(i)-(i i)$, and $\sigma_{H_{3}}$ in Configuration (iii)). In contrast, distortions in the $\Omega$ and $\Gamma$ functions play a crucial role for indeterminacy. In fact, although Assumption 5 cannot be verified and indeterminacy is not possible with only arbitrarily small distortions on $\varrho$ (see Proposition 4 ), this is no longer the case when small distortions influence $\Omega$, or $\Gamma$, or both. Indeed, in the absence of distortions on $\varrho$, Assumption 5 is satisfied with (arbitrarily) small values of $\alpha_{L, L}, \alpha_{\Gamma, L}, \alpha_{\Gamma, K}$ and $\alpha_{L, K}$, provided they are such that $\alpha_{\Gamma, K}-$ $\alpha_{L, K} \leq 0, \alpha_{L, L}>0$ and/or $\alpha_{\Gamma, L}<0$ and, therefore, provided also that $\alpha_{L, L}-$ $\alpha_{\Gamma, L}$ is small and positive. ${ }^{17}$ Applying Proposition 5 and using Corollary 3 (condition $i$ ), we obtain configuration $(i)$. Hence, indeterminacy occurs when $\sigma$ exceeds $\sigma_{H_{1}}$ and for $\epsilon_{\gamma}$ below $\epsilon_{\gamma H}$ or $\epsilon_{\gamma T}$. However, when distortions become arbitrarily small, ${ }^{18} \sigma_{H_{1}} \rightarrow+\infty$ (see (18), so that indeterminacy requires an arbitrarily large $\sigma$. In this case $\varepsilon_{\gamma_{H}} \rightarrow 1$ and $\varepsilon_{\gamma T} \rightarrow 1$ (see (21), (23) in Appendix 6.3.3 and 6.3.4) and the following result is obtained.

Corollary 4 Assume that Proposition 5 applies. Indeterminacy occurs with arbitrarily small distortions in $\Omega$ and/or $\Gamma$, if and only if $\alpha_{\Gamma, K}-\alpha_{L, K} \leq 0$, and either $\alpha_{L, L}>0$ or $\alpha_{\Gamma, L}<0$, and the elasticity of capital-labor substitution and the elasticity of private labor supply are arbitrarily large.

\footnotetext{
${ }^{17}$ All the other Assumptions of Proposition 5 are also verified when we only have arbitrarily small distortions in $\Omega$ and/or $\Gamma$.

${ }^{18}$ Note that in this case $\alpha_{\Gamma, L} \rightarrow 0, \alpha_{\Gamma, L}^{*} \rightarrow 0, \alpha_{\Gamma, L}^{H} \rightarrow 0$ and, under Assumption 5, $\alpha_{\Gamma, L}^{*}-\alpha_{\Gamma, L} \rightarrow 0^{+}$and $\alpha_{\Gamma, L}^{H}-\alpha_{\Gamma, L} \rightarrow 0^{+}$.
} 
This corollary, together with Proposition 4 suggests that a minimal degree of distortions is required for indeterminacy to occur with plausible values of the elasticity of substitution between labor and capital. In Section 4 we discuss this minimal degree of distortions for each example considered.

Another conclusion worth emphasizing is that, while distortions on $\Omega$ and $\Gamma$ seem to play a similar (symmetric) role on indeterminacy when they are small, this is no longer the case when these distortions take higher values. Indeed, (non arbitrarily small) distortions on $\Gamma$ play a crucial role for the occurrence of flip bifurcations. From Corollary 3 we see that in the absence of distortions on $\Gamma$ we would obtain, for credible calibrations of the parameters (low $\theta$ ), mainly configuration $(i)$ where those bifurcations are ruled out. Hence, since flip bifurcations are frequently a route for chaos, distortions affecting the offer curve may be associated with complex chaotic behavior of capital and labor/employment trajectories.

Finally, two remarks concerning the scope of our results are worth referring. First, our paper only deals with the role of market distortions on local indeterminacy linked to the sink property, i.e. we do not address the cases of static or global dynamic indeterminacy and bifurcations, which may also appear in the presence of some market imperfections. ${ }^{19}$ Second, although we only discuss local deterministic indeterminacy and bifurcations, associated with the emergence of deterministic or cycles, we may construct stochastic sunspot equilibria, i.e. expectation driven fluctuations, along indeterminacy and/or flip/Hopf bifurcations, ${ }^{20}$ as shown in Grandmont et al. (1998). ${ }^{21}$

\section{Applications}

We now present several examples of specific distortions that provide microeconomic foundations for the model developed above. Many of them have already been studied in the literature, but not always in a finance constrained Woodford economy. In Section 4.1 we describe the examples and we repre-

\footnotetext{
${ }^{19}$ Static indeterminacy is considered by Dos Santos Ferreira and Dufourt (2006), Dos Santos Ferreira and Lloyd-Braga (2008) and Wang and Wen (2008) to explain expectationdriven fluctuations. Drugeon and Wigniolle (1996) and Gali (1995) obtain multiplicities from the analysis of global dynamics.

${ }^{20}$ Note that sunspots cycles obtained along Hopf or flip bifurcations, with characteristic roots of the linearized system close to one, will tend to exhibit endogenous persistency, a feature present in real data.

${ }^{21}$ However, under some specific market failures, with strategic interactions between agents, our parameters $\alpha_{i j}$ and $\beta_{i j}$ may become stochastic in the presence of extrinsic uncertainty and, in this case, the results provided in Grandmont et al. (1998) may no longer be applicable.
} 
sent them according to our general approach, showing that some of them have equivalent representations. In Section 4.2 we apply our results to understand in which cases indeterminacy can occur for plausible values of the elasticity of capital-labor substitution.

\subsection{Specific Distortions and Equivalence Results}

For each example, we start by identifying the $\varrho(K, L), \Omega(K, L)$ and $\Gamma(K, L)$ functions. We then compute the elasticities of these three functions with respect to $K$ and $L$, evaluated at the steady state and, using (6), we identify the parameters $\alpha_{i j}$ and $\beta_{i j}$ for $i=K, L, \Gamma$ and $j=K, L$ as functions of parameters that represent the specific distortions in each example. ${ }^{22}$

We also emphasize that there are classes of specific distortions which have equivalent representations in terms of our distortions parameters. Since market distortions influence local dynamics through the parameters $\alpha_{i j}$ and $\beta_{i j}$, equivalent market distortions share the same local dynamic properties and indeterminacy mechanisms, even if their economic interpretations are different. Our equivalence results have strong implications. If we estimate the relevant parameters of our general formulation, we will not be able to identify a particular source of specific distortions among those, which belonging to the same class, are observational equivalent. Also, even if indeterminacy requires an empirically unreasonable degree of some specific distortion, the associated indeterminacy mechanism is not necessarily unimportant, since an equivalent empirically plausible model may exist. Another implication is that simulations of equivalent linearized versions of the model with additive shocks lead exactly to the same trajectories of aggregate capital and labor (in deviations from the steady state) and, thereby, equilibrium cyclical properties of variables that only depend on aggregate capital and labor are, up to the first order, identical in equivalent models. Finally, the dynamic effects of one specific distortion may be compensated/eliminated by the existence of another symmetric specific distortion belonging to the same equivalence class. This last result suggests some policy implications. Indeed, as we shall see below in Section 4.2.2, some forms of taxation eliminate local indeterminacy and endogenous fluctuations caused by the presence of consumption externalities, so that distortionary taxes may be defended on stability grounds.

\footnotetext{
${ }^{22}$ In all the examples, $s, \sigma, \varepsilon_{\gamma}, \gamma(L), \omega\left(K_{t-1} / L_{t}\right), \rho\left(K_{t-1} / L_{t}\right)$ and $A F\left(K_{t-1}, L_{t}\right)$ are defined as in Section 2.1.
} 


\subsubsection{Examples with the same distortion on the real interest rate and the real wage}

Here we explore examples of output market distortions. In these examples, the generalized offer curve coincides with the competitive one, $\Gamma(K, L)=$ $\gamma(L)$, and the real interest rate and the real wage are affected in the same way, i.e. we have:

$$
\begin{aligned}
\Omega_{t} & =\omega_{t} D\left(K_{t-1}, L_{t}\right)=A \omega\left(K_{t-1} / L_{t}\right) D\left(K_{t-1}, L_{t}\right) \\
\varrho_{t} & =\rho_{t} D\left(K_{t-1}, L_{t}\right)=A \rho\left(K_{t-1} / L_{t}\right) D\left(K_{t-1}, L_{t}\right)
\end{aligned}
$$

where $D(K, L)$ stands for the distortion introduced, so that $\varepsilon_{\Omega, K}=\varepsilon_{D, K}+\frac{s}{\sigma}$, $\varepsilon_{\Omega, L}=\varepsilon_{D, L}-\frac{s}{\sigma}, \varepsilon_{\varrho, K}=\varepsilon_{D, K}-\frac{1-s}{\sigma}, \varepsilon_{\varrho, L}=\varepsilon_{D, L}+\frac{1-s}{\sigma}$. Using (6) the parameters $\alpha_{i j}$ and $\beta_{i j}$ for $i=K, L$ and $j=K, L$ are easily obtained.

Productive Externalities Productive externalities have often been introduced in macro-dynamic models (Barinci and Chéron (2001), Benhabib and Farmer (1994), Cazzavillan (2001), Cazzavillan et al. (1998)). In these papers all markets are perfectly competitive and firms face a private constant returns to scale technology, but, due to positive externalities that affect the total productivity of factors, returns to scale are increasing at the social level. Here, we will extend this formulation, allowing also for negative productive externalities so that, at the social level, returns to scale can be decreasing. We consider therefore that production is given by $y=A F(K, L) \xi(\bar{K}, \bar{L})$, where $\xi(\bar{K}, \bar{L})$ stands for externalities, $\bar{K}(\overline{L)}$ denoting average levels of capital (labor). Since firms, when maximizing profits, take externalities as given, at a symmetric equilibrium with $\bar{K}=K$ and $\bar{L}=L$, we have $D\left(K_{t-1}, L_{t}\right)=\xi\left(K_{t-1}, L_{t}\right)$. Denoting by $\varepsilon_{\xi, i}$ the elasticity of the function $\xi(K, L)$ with respect to $i=K, L$, evaluated at the steady state, we obtain $\alpha_{L, L}=\alpha_{K, L}=\varepsilon_{\xi, L}, \alpha_{L, K}=\alpha_{K, K}=\varepsilon_{\xi, K}$, and $\alpha_{\Gamma, i}=\beta_{j, i}=0, i=K, L$ and $j=K, L, \Gamma$.

Output externalities can also be represented in this framework if we consider $\xi(\bar{K}, \bar{L})=Z(\overline{F(K, L)})$, where $\overline{F(K, L)}$ is the average private level of output. Defining the elasticity of $Z$ with respect to $F, z \equiv \varepsilon_{Z, F}$, we obtain $\alpha_{L, L}=\alpha_{K, L}=(1-s) z, \alpha_{L, K}=\alpha_{K, K}=s z$. We can see that output externalities are a particular case of positive productive externalities, generating the same values for $\alpha_{i j}, \beta_{i j}$ when $\varepsilon_{\xi, L}$ and $\varepsilon_{\xi, K}$ are such that $z=\varepsilon_{\xi, L}+\varepsilon_{\xi, K}$ with $\varepsilon_{\xi, L} / \varepsilon_{\xi, K}=(1-s) / s$.

Imperfect competition in the product market We will now emphasize that, from a local dynamics point of view, many models with imperfect 
competition in the product market are, in fact, a particular case of the previous framework with positive productive externalities, corresponding more precisely to the case of output externalities. Benhabib and Farmer (1994) and Cazzavillan, Lloyd-Braga and Pintus (1998) underlined that this is the case when imperfectly competitive economies are characterized by the existence of firms that have internal increasing returns (the private production function being homogeneous in $K$ and $L$ of degree $1+z>1$ ) associated with decreasing marginal costs (the cost function being homogeneous of degree $1 /(1+z)<1$ in output). However, in these models, the equilibrium markup is constant, while several empirical studies (Bils (1987), Martins and Scarpetta (1999), Portier (1995), Rotemberg and Woodford (1991)) have shown that markups are typically countercyclical. Here, we show that the same type of equivalence exists with models of imperfect competition where markup variability is linked to strategic interactions between producers and business formation. In these models increasing returns are associated with a fixed cost, each typical firm $i=1, \ldots, N_{t}$ producing according to $A\left[F\left(k_{i t-1}, l_{i t}\right)-\phi\right]$, where $k_{i t-1}\left(l_{i t}\right)$ represents capital (labor) used by firm $i$ and $\phi>0$ is a fixed cost. For the sake of exposition, we focus here on markup variability applied in the context of Cournot competition under free entry (where also the obtained elasticity of output market demand, $\tau$, is constant, as for instance in Seegmuller (2003) and Dos Santos Ferreira and Lloyd-Braga (2005)). The number $N_{t}$ of producers is determined by the usual zero profit condition and the markup factor, at a symmetric equilibrium, is given by $\mu_{t}=\mu\left(N_{t}\right) \equiv \frac{\tau N_{t}}{\tau N_{t}-1}$ with $\epsilon_{\mu}(N) \equiv \mu^{\prime}(N) N / \mu(N)<0$. Firms, maximizing profits, choose capital (and labor) such that the ratio of the marginal productivity of capital (labor) over the markup factor equals the real interest rate (wage). At equilibrium, the number of firms is procyclical, i.e. it is an increasing function of individual (and aggregate) production, and can be written as a function of aggregate capital $K=N k$ and labor $L=N l$, i.e., $N_{t}=N\left(Y_{t}\right)=\sqrt{\frac{Y_{t}}{\phi \tau}}$ with $Y_{t} \equiv F\left(K_{t-1}, L_{t}\right)$ and $\epsilon_{N}(Y) \equiv N^{\prime}(Y) Y / N(Y)>0$. As a result the markup is countercyclical, and the same distortion $D\left(K_{t-1}, L_{t}\right)=1 / \mu\left(N\left(K_{t-1}, L_{t}\right)\right)$ affects both the real wage and the real interest rate. Defining $\nu \equiv-\epsilon_{\mu}(N) \epsilon_{N}(Y)>0$, we obtain, $\alpha_{L, L}=\alpha_{K, L}=(1-s) \nu, \alpha_{L, K}=\alpha_{K, K}=s \nu$ and $\alpha_{\Gamma, i}=\beta_{j, i}=0$, for $i=K, L$ and $j=K, L, \Gamma$. Comparing with positive productive externalities, with output externalities and with models of constant markup, we can state the following result.

Proposition 6 Models with imperfect competition in the product market with countercyclical markup variability (characterized by $\nu>0$ ), as described above, have equivalent representation, in terms of our general framework, 
to models with positive productive externalities $\left(\varepsilon_{\xi, L}>0, \varepsilon_{\xi, K}>0\right)$ when $\nu=\varepsilon_{\xi, L}+\varepsilon_{\xi, K}$ with $\varepsilon_{\xi, K} / \varepsilon_{\xi, L}=s /(1-s)$, and have equivalent representation to models with positive output externalities or to models of constant markup (characterized by $z>0$ ) when $\nu=z$.

In fact, this equivalence can be extended to several other types of imperfectly competitive output markets with markup variability. The equivalence applies, for instance, under free entry with monopolistic competition where aggregate consumption (see Seegmuller (2009) or aggregate output (see Dos Santos Ferreira and Lloyd-Braga (2005), Kuhry (2001), Weder (2000a))) is given by a function à la Dixit-Stiglitz in several differentiated goods, each good being produced under a fixed cost as described above. It also applies to models with taste for variety defined as, following Benassy (1996), the consumer utility gain of consuming one unit of all the $N_{t}$ available varieties of goods instead of consuming $N_{t}$ units of a single variety (Jacobsen (1998), Seegmuller (2008)). However, in this case $\mu$ represents the ratio between the aggregate price and the price set by a single firm, instead of representing the markup factor. Of course, the precise functional forms of $\mu\left(N_{t}\right), \epsilon_{\mu}(N)$, $N\left(Y_{t}\right)$ and $\epsilon_{N}(Y)$ depend on the specific model considered.

Finally let us remark that this equivalence must also hold in several different types of macrodynamic models and not only in the Woodford framework, as long as capital and labor demand are derived from (static) profit maximization as in our model.

\subsubsection{Examples with different distortions on the real interest rate and effective consumption}

The examples considered here are capital market distortions (capital income taxation), labor market distortions (labor income taxation) and output market distortions (consumption externalities). These distortions still do not affect the generalized offer curve $(\Gamma(K, L)=\gamma(L))$, but now the real interest rate and effective consumption are not affected in the same way, i.e. we have:

$$
\begin{aligned}
\Omega_{t} & =\omega_{t} D_{1}\left(K_{t-1}, L_{t}\right)=A \omega\left(K_{t-1} / L_{t}\right) D_{1}\left(K_{t-1}, L_{t}\right) \\
\varrho_{t} & =\rho_{t} D_{2}\left(K_{t-1}, L_{t}\right)=A \rho\left(K_{t-1} / L_{t}\right) D_{2}\left(K_{t-1}, L_{t}\right)
\end{aligned}
$$

so that $\varepsilon_{\Omega, K}=\varepsilon_{D_{1}, K}+\frac{s}{\sigma}, \varepsilon_{\Omega, L}=\varepsilon_{D_{1}, L}-\frac{s}{\sigma}, \varepsilon_{\varrho, K}=\varepsilon_{D_{2}, K}-\frac{1-s}{\sigma}, \varepsilon_{\varrho, L}=$ $\varepsilon_{D_{2}, L}+\frac{1-s}{\sigma}$. See $(6)$.

Public spending financed by variable taxation In this example we consider a perfectly competitive economy where public expenditures, financed 
by variable taxation under a balanced budget rule, are introduced. This example follows closely, although extending it by also considering capital income taxation, the work of Lloyd-Braga et al. (2008), and covers as particular cases the fiscal policy rules considered in Dromel and Pintus (2008), Guo and Lansing (1998), Gokan (2005), Pintus (2003), Schmitt-Grohé and Uribe (1997), among others. The government can levy taxes on capital income $\left(\rho_{t} K_{t-1}\right)$ and labor income $\left(\omega_{t} L_{t}\right)$. Real public spending in goods and services in period $t, G_{t} \geq 0$, is given by the balanced budget rule $G_{t}=\tau_{L}\left(\omega_{t} L_{t}\right) \omega_{t} L_{t}+\tau_{K}\left(\rho_{t} K_{t-1}\right) \rho_{t} K_{t-1}$. Tax rates on labor and capital incomes are determined respectively by the fiscal policy rules $\tau_{L}\left(\omega_{t} L_{t}\right) \equiv$ $z_{L}\left(\omega_{t} L_{t} / \omega L\right)^{\phi_{L}}$ and $\tau_{K}\left(\rho_{t} K_{t-1}\right) \equiv z_{K}\left(\rho_{t} K_{t-1} / \rho K\right)^{\phi_{K}}$, with parameters $z_{i} \in$ $(0,1)$ and $\phi_{i} \in R$ for $i=L, K$, and where $\omega L$ and $\rho K$ are respectively the wage bill and capital income, both evaluated at the steady state. Note that $z_{i}$ represents the tax rate at the steady state and that $\phi_{i}$ denotes the elasticity of the tax rate with respect to the tax base. When $\phi_{i}=0$ the tax rate is constant at the level $z_{i}$. Since the unique difference with respect to the perfectly competitive economy is that now the real wage received by workers is given by $\omega_{t}\left[1-z_{L}\left(\omega_{t} L_{t} / \omega L\right)^{\phi_{L}}\right]$ instead of $\omega_{t}$ and the real interest rate received by capitalists is given by $\rho_{t}\left[1-z_{K}\left(\rho_{t} K_{t-1} / \rho K\right)^{\phi_{K}}\right]$ instead of $\rho_{t}$, we obtain:

$$
\begin{aligned}
& D_{1}\left(K_{t-1}, L_{t}\right)=1-z_{L}\left(\omega_{t} L_{t} / \omega L\right)^{\phi_{L}} \\
& D_{2}\left(K_{t-1}, L_{t}\right)=1-z_{K}\left(\rho_{t} K_{t-1} / \rho K\right)^{\phi_{K}}
\end{aligned}
$$

In the following, we will address separately each type of taxation.

In the case of capital taxation market distortions only appear in the function $\varrho(K, L)$, i.e. $D_{1}(K, L)=1$. We get $\alpha_{j, i}=\beta_{j, i}=0$ for $i=K, L$ and $j=L, \Gamma, \alpha_{K, K}=-\phi_{K} \frac{z_{K}}{1-z_{K}}, \alpha_{K, L}=0$ and $\beta_{K, K}=-\alpha_{K, K}(1-s)=-\beta_{K, L}$.

In the case of labor income taxation distortions only affect effective consumption $\Omega(K, L)$, i.e. $D_{2}(K, L)=1$. We have $\alpha_{j, i}=\beta_{j, i}=0$ for $i=K, L$ and $j=K, \Gamma, \alpha_{L, L}=-\phi_{L} \frac{z_{L}}{1-z_{L}}, \alpha_{L, K}=0$ and $\beta_{L, L}=-\alpha_{L, L} s=-\beta_{L, K}$.

Consumption externalities Consumption externalities correspond to the idea that the individual utility of consumption is affected by the current consumption of others (envy or altruism), so that aggregate or average consumption becomes an argument of the utility function (Alonso-Carrera et al. (2008), Gali (1994), Ljungqvist and Uhlig (2000), Weder (2000b)). Here, we consider that individual workers compare their own consumption to that of the average worker, so that workers' utility is given by $U\left(C_{t+1}^{w} \varphi\left(\bar{C}_{t+1}^{w}\right) / B\right)-$ $V\left(L_{t}\right)$, where $\bar{C}_{t+1}^{w}$ denotes workers' average consumption and $\varphi\left(\bar{C}_{t+1}^{w}\right)$ the 
externality function. Accordingly, at equilibrium we have $\bar{C}^{w}=C^{w}$ and $D_{1}\left(K_{t-1}, L_{t}\right)=\varphi\left(C_{t}^{w}\right)$. Denoting by $\chi$ be the elasticity of $\varphi$ with respect to $\bar{C}^{w}$, evaluated at the steady state, we have $\alpha_{L, L}=\chi, \alpha_{L, K}=0, \beta_{L, L}=$ $-\alpha_{L, L} s=-\beta_{L, K}$. Comparing with the example on labor taxation, we have the following result.

Proposition 7 Models with consumption externalities in preferences where individual workers compare their own consumption to that of the average worker (characterized by $\chi$ ) and models with labor income taxation $\left(\phi_{L}, z_{L}\right)$ described above, have equivalent representations in terms of our general framework when $\chi=-\phi_{L} \frac{z_{L}}{1-z_{L}}$.

Note that, when individual workers compare their own consumption to that of the average consumer, we have an equivalence with models with consumption taxation of the type proposed by Lloyd-Braga et al. (2008).

\subsubsection{Examples with distortions on the generalized offer curve}

We start with examples where distortions only modify the offer curve (leisure externalities and unemployment insurance with efficiency wages) and proceed with an example where effective consumption is also affected (unemployment benefits and unions).

Leisure externalities The idea behind leisure externalities is that an individual's utility from leisure (or disutility of labor) is affected by the amount of leisure consumed (or labor supplied) by others. For the sake of simplicity, let the utility function of a worker be written as $C_{t+1}^{w} / B-\bar{L}_{t}^{\mu} L_{t}^{\epsilon_{\gamma}}$, where $\epsilon_{\gamma} \geq 1$ corresponds to the elasticity of the offer curve in the absence of distortions, $\mu \in \mathbb{R}$ is a constant parameter and $\bar{L}_{t}$ denotes aggregate labor, taken as given by individual workers, but modifying their welfare. Since at equilibrium $L_{t}=\bar{L}_{t}$, the generalized offer curve becomes $\Gamma(K, L)=L^{\epsilon_{\gamma}+\mu}$. Solving the model we get $\alpha_{j i}=\beta_{j i}=0$ for $\{i, j\}=\{K, L\}, \beta_{\Gamma L}=-\beta_{\Gamma K}=$ 0 and $\alpha_{\Gamma L}=\mu$. This example covers, as particular cases, the type of leisure externalities considered by Benhabib and Farmer (2000) and Weder (2004), where $\mu<0$, implying that the (private marginal) desutility of labor is lower when others also work more. Note that in this case the elasticity of the generalized offer curve becomes lower than $\epsilon_{\gamma}$ and therefore it can take values lower than 1 , in contrast to the perfectly competitive case. If, for instance, $\varepsilon_{\gamma}=1$ and $\mu<0$ we obtain $\varepsilon_{\Gamma L}<1$, as it happens in the model explored in Grandmont (2008) that we present below. 
Unemployment insurance and efficiency wages Grandmont (2008) introduces unemployment insurance in a Woodford economy with efficiency wages (see also Coimbra (1999) and Nakajima (2006)), distorting only the generalized offer curve. There is a continuum of identical workers of mass 1 , with disutility from effort, labor is indivisible and unemployed workers receive a constant percentage $z$ of the wage, financed by a uniform tax rate on income of all workers, both, the benefits and the tax rate, taken as given by individuals. The efficiency wage contracting involves a level of effort, $x^{*}$, and a level of consumption of employed workers, $C^{*}$, both constant over time (depending only on $z$ ). Therefore, there is a constant reservation wage at the private level, so that $\varepsilon_{\gamma}=1$. In this model, $\Gamma(K, L)$ is identical to aggregate consumption of employed and unemployed workers, i.e., $\Gamma(K, L)=$ $C^{*} n+C^{*}(1-n) z$, where $n=L / x^{*}$ denotes the steady state employment rate. We get $\varepsilon_{\Gamma K}=0$ and $0<\varepsilon_{\Gamma L}=\frac{(1-z) n}{z+(1-z) n}<1$. Hence, $\alpha_{\Gamma L}=\frac{-z}{z+(1-z) n}$, $\alpha_{\Gamma L}=\beta_{\Gamma L}=\beta_{\Gamma K}=0, \alpha_{j i}=\beta_{j i}=0$ for $\{i, j\}=\{K, L\}$. Comparing this economy with the one with leisure externalities we can state the following:

Proposition 8 Assuming $\varepsilon_{\gamma}=1$, the model with unemployment benefits and efficiency wages (characterized by $1>z>0, n>0$ ) and the model with externalities in leisure $(\mu<0)$, described above, have equivalent representations in terms of our general framework when $-1<\frac{-z}{z+(1-z) n}=\mu<0$.

Unions and unemployment benefits We end this section presenting an example with labor market imperfections, where both $\Gamma(K, L)$ and $\Omega(K, L)$ are affected by market distortions. This example is provided by the model developed in Dufourt et al. (2008) that introduces unions and unemployment benefits in the Woodford finance constrained framework (see also LloydBraga and Modesto (2007)). There is a continuum of identical workers, labor is indivisible and workers have no labor desutility. A constant real benefit $b>0$ is paid to each unemployed in $t$ financed by a real tax $\tau>0$ on each employed worker at $t$, both being received and paid at $t+1$, i.e., $b(1-L)=\tau L$. Firms first decide the amount of capital services to rent and then wages and employment are determined through an efficient bargain between unions and firms. Agents take $b$ and $\tau$ as given, and unions, willing to maximize the consumption of an average worker, are able to set wages paid by firms above a reservation wage $\overline{R W}$ (given by $\overline{R W_{t}}=(b+\tau) \frac{p_{t+1}}{p_{t}}$ ), with a markup factor $\mu(K, L)=\frac{1-\alpha s(K, L)}{1-s(K, L)} \geq 1$, increasing in the (constant) bargaining power of unions $(1-\alpha) \in[0,1)$. Employment is determined by the equality between the reservation wage and the marginal productivity of labor, i.e., $\overline{R W}_{t}=\omega\left(K_{t-1} / L_{t}\right)$. Note that the case of a perfectly competitive 
labor market would be obtained with $\alpha=1$ leading to $\mu(K, L)=1$. Capital is determined by the equality between the real interest rate and the marginal productivity of capital multiplied by the firms' bargaining power, $\alpha$. Recall that money demand in every period $t$ is identical to the respective wage bill $\left(\mu\left(K_{t-1}, L_{t}\right) \omega\left(K_{t-1} / L_{t}\right) L_{t}\right)$ and money supply is constant over time. From monetary equilibrium we obtain $\frac{p_{t+1}}{p_{t}}=\frac{\left.\mu\left(K_{t-1}, L_{t}\right) \omega\left(K_{t-1} / L_{t}\right) L_{t}\right)}{\left.\mu\left(K_{t}, L_{t+1}\right) \omega\left(K_{t} / L_{t+1}\right) L_{t+1}\right)}$. Hence, given the equations that determine real wages, employment and real interest rates, we are able to identify this model within our general framework, obtaining:

$$
\begin{aligned}
\Omega\left(K_{t-1}, L_{t}\right) & =\mu\left(K_{t-1} / L_{t}\right) A \omega\left(K_{t-1} / L_{t}\right) \\
\varrho\left(K_{t-1}, L_{t}\right) & =A \alpha \rho\left(K_{t-1} / L_{t}\right) \\
\Gamma\left(K_{t-1}, L_{t}\right) & =b \mu\left(K_{t-1} / L_{t}\right)
\end{aligned}
$$

Because of the existence of a constant reservation wage at the individual level, we have $\epsilon_{\gamma}=1$ and, since $\varepsilon_{\mu, K}=-\varepsilon_{\mu, L}=\frac{s(1-s)}{1-\alpha s} \frac{(\sigma-1)}{\sigma}$, after some computations we get $\alpha_{K, j}=\beta_{K, j}=0$ for $j=K, L,{ }^{23} \alpha_{\Gamma, K}=\alpha_{L, K}=-\alpha_{L, L}=$ $-\beta_{\Gamma, K}=\beta_{L, L}=-\beta_{L, K}=\beta_{\Gamma, L}=\frac{s(1-\alpha)}{1-\alpha s} \in(0, s)$, and $\alpha_{\Gamma, L}=\alpha_{L, L}-1$.

\subsection{Discussing the Examples: The minimal degree of distortions required for indeterminacy}

In this section we apply the results obtained in Proposition 5 to the examples presented in the preceding section. In Table 2 we summarize the assumptions and configurations obtained in terms of parameters that represent the specific distortions considered in each example, focusing mainly on Assumptions 1 and $5 .^{24}$ This table only covers the basic examples, the others being easily recovered using our equivalence Propositions.

As referred in Section 3.3, a minimal degree of distortions is required for the occurrence of indeterminacy with plausible values for the elasticity of substitution between capital and labor. Here, using Table 2 and Proposition

\footnotetext{
${ }^{23}$ This means that, in terms of local dynamics, the model is as if $\varrho\left(K_{t-1}, L_{t}\right)$ is not affected by distortions.

${ }^{24}$ In all the examples presented, Assumptions 2 and 3 are always satisfied. Also, under Assumption 1, Assumption 4 is verified in all examples, except in the case of leisure externalities where it requires a not too negative degree of externalities. It can also be checked that, under Assumptions 1 and 4, Assumption 6 is also satisfied in all the examples. Note also that Assumptions 7 and 8 only apply in the case of output market imperfections, and in particular in the case of productive externalities presented in Table 2, where $\alpha_{K, K}>0$. Finally, Assumption 9 only applies in the case of labor market imperfections (in particular in the case of leisure externalities and unemployment benefits with unions) where Configuration (iii) exists, being always satisfied in those examples.
} 


\begin{tabular}{|c|c|c|c|}
\hline Parameters & Assumption 1 & Assumption 5 & Configurations obtained \\
\hline & $\theta(1-s)<s<\frac{1}{2}$ & & \\
\hline $\begin{array}{l}\text { Productive Externalities } \\
\varepsilon_{\xi, L}=\alpha_{L, L}=\alpha_{K, L} \\
\varepsilon_{\xi, K}=\alpha_{L, K}=\alpha_{K, K}\end{array}$ & $\begin{array}{l}\varepsilon_{\xi, L}>-1 \\
\varepsilon_{\xi, K}>-\frac{1}{\theta} \\
\theta\left[\left(1+\varepsilon_{\xi, L}\right)(1-s)\right. \\
\left.-s \varepsilon_{\xi, K}\right]<s \\
\sigma>\frac{s}{1+\varepsilon_{\xi, L}}\end{array}$ & $\begin{array}{l}\varepsilon_{\xi, L}>\theta \varepsilon_{\xi, K} \\
\varepsilon_{\xi, L}+\varepsilon_{\xi, K}>0 \\
\text { Ass. } 7 \text { requires } \\
\varepsilon_{\xi, L}>\frac{s}{1-s} \varepsilon_{\xi, K}\end{array}$ & $\begin{array}{l}\text { Extending Ass. } 1 \text { to } \\
\varepsilon_{\xi, L}>0, \varepsilon_{\xi, K}>0 ; \\
\theta\left[\left(1+\varepsilon_{\xi, L}\right)\left(1-s+\varepsilon_{\xi, L}\right.\right. \\
\left.\left.+\varepsilon_{\xi, K}\right)-s \varepsilon_{\xi, K}\right]<s \\
\text { we obtain configuration (i) } \\
\text { and Ass. } 8 \text { is satisfied }\end{array}$ \\
\hline $\begin{array}{l}\text { Labor Taxation } \\
0<z_{L}<1 ; \phi_{L} \\
\alpha_{L, L}=-\phi_{L} \frac{z_{L}}{1-z_{L}} \\
\beta_{L, L}=-\beta_{L, K}=-\alpha_{L, L} s\end{array}$ & $\begin{array}{l}-1<\phi_{L} \frac{z_{L}}{1-z_{L}}<1 \\
\sigma>s\end{array}$ & $\begin{array}{l}\phi_{L} \frac{z_{L}}{1-z_{L}}<0 \\
\text { implying } \phi_{L}<0\end{array}$ & $\begin{array}{l}\text { Extending Ass. } 1 \text { to } \\
3 \theta(1-s)<2 s \\
\text { we obtain configuration (i) }\end{array}$ \\
\hline $\begin{array}{l}\text { Leisure Externalities } \\
\mu=\alpha_{\Gamma, L}\end{array}$ & $\sigma>s$ & $\mu<0$ & $\begin{array}{l}\text { As Ass. } 4 \text { implies } \mu>-1 \\
\text { we obtain configuration: } \\
\text { (i) for } \alpha_{\Gamma L}^{b}<\mu<0, \\
\text { (ii) for } \alpha_{\Gamma L}^{c}<\mu<\alpha_{\Gamma L}^{b}, \\
\text { (iii ) for }-1<\mu<\alpha_{\Gamma, L}^{c}, \\
\alpha_{\Gamma L}^{b} \equiv-1+\frac{\theta(1-s)}{2 s-\theta(1-s)} \\
\alpha_{\Gamma L}^{c} \equiv \frac{2[s-\theta(1-s)]}{\theta(1-s)} \\
-\frac{2 \sqrt{s[s-\theta(1-s)]]}}{\theta(1-s)}\end{array}$ \\
\hline $\begin{array}{l}\text { Unemployment benefits } \\
\underline{\text { and unions }} \\
0<\alpha \leq 1, \varepsilon_{\gamma}=1 \\
\alpha_{L, L}=-\frac{s(1-\alpha)}{1-\alpha s} \\
\alpha_{\Gamma, L}=\alpha_{L, L}-1 \\
\alpha_{i, K}=-\alpha_{L, L} \\
\beta_{i, L}=-\beta_{i, K}=-\alpha_{L, L} \\
\text { for } i=\Gamma, L\end{array}$ & $\begin{array}{l}\theta(1-s)<\alpha s \\
\sigma>\alpha s\end{array}$ & & $\begin{array}{l}\text { We obtain configurations } \\
\text { (i ) and (ii) } \\
\text { for } \frac{\theta(1-s)}{s}<\alpha<\alpha^{*} \\
\text { and configuration (iii) } \\
\text { for } \alpha^{*}<\alpha \leq 1 \text {, where } \\
\alpha^{*} \equiv 1-\frac{\theta(1-s)}{s(4-\theta)} \text {. }\end{array}$ \\
\hline
\end{tabular}

Table 2: Assumptions and configurations expressed in terms of the parameters of the specific examples 
5, we discuss this minimal degree of distortions, focusing particularly in the case of a Cobb Douglas technology $(\sigma=1)$. Our numerical examples are obtained considering that $\beta=(1 / 1.03)^{\frac{1}{4}}$ and $\delta=0.1 / 4$, which is consistent with most calibrations used in the business cycle literature for quarterly data. Hence, $\theta=0.03475$, and we fix $s=0.35$, so that $\theta(1-s)<s<$ 0.5, as required by Assumption 1. We organize our discussion grouping the different examples according to the effectiveness of the specific distortions on indeterminacy.

\subsubsection{Distortions that affect the real interest rate but not the generalized offer curve}

In these examples, as distortions do not affect the offer curve, by Corollary 1(ii), under Assumption 1 indeterminacy always requires Assumption 5.1.

In the case of productive externalities, indeterminacy cannot occur if externalities are negative $\left(\varepsilon_{\xi, L}<0, \varepsilon_{\xi, K}<0\right)$. Indeed, in this case $\alpha_{K K}=\varepsilon_{\xi, K}<0$ and by Corollary 2(i) Assumption 5.2 is also required for indeterminacy. However, this Assumption $\left(\varepsilon_{\xi, L}+\varepsilon_{\xi, K}>0\right)$ cannot be satisfied when $\varepsilon_{\xi, L}<0, \varepsilon_{\xi, K}<0$. With positive externalities ${ }^{25}$, as in Cazzavillan et al. (1998), Configuration ( $i$ ) of Table 1 applies (see Table 2) and indeterminacy can emerge for $\sigma>\sigma_{H_{1}}=(s-\theta(1-s)) /\left(\varepsilon_{\xi, L}+\varepsilon_{\xi, K}-(1+\theta) \varepsilon_{\xi, K}\right)$. Hence, indeterminacy can only emerge in the Cobb-Douglas case with $\varepsilon_{\xi, L}+\varepsilon_{\xi, K}>$ $[s-\theta(1-s)]+(1+\theta) \varepsilon_{\xi, K}>[s-\theta(1-s)]=0.3274125$, under our calibration. This is a high value, hardly reconcilable with empirical studies. See, for instance, Basu and Fernald (1997) that found degrees of increasing returns between 0.03 and 0.18 for the U.S. economy, or Harrison (2003) who finds no significant externalities for the U.S. manufacturing industry at the 2 digits level.

With markup variability we may apply the equivalence result of Proposition 6 . Therefore, $\nu \equiv-\epsilon_{\mu}(N) \epsilon_{N}(Y)$ should be high enough to get indeterminacy with a value of $\sigma$ close to 1 . In the case of Cournot competition, where $\epsilon_{\mu}(N)=1-\mu$ and $\epsilon_{N}(Y)=1 / 2$, we should have $\mu>1+2 \frac{s-\theta(1-s)}{(1-s)-\theta s}=1.95$ under our quarterly calibration (or $\mu>1.853$ with an annual calibration), which exceeds empirical estimates. See, for instance, Morrison (1993) where the estimated average annual markups of U.S. manufacturing industries are between 1.179 and 1.803 .

With capital taxation, easy computations show that Assumption 5.1 implies that $\alpha_{K K}<0$, i.e. $\phi_{K}>0$, so that tax rates vary positively with

\footnotetext{
${ }^{25}$ The reader may check that with positive externalities $\left(\varepsilon_{\xi, L}>0\right.$ and $\left.\alpha_{K K}=\varepsilon_{\xi, K}>0\right)$, we have $\alpha_{\Gamma L}^{*}>\alpha_{\Gamma L}^{H}$, so that (24) is satisfied and Assumption 8 is verified.
} 
capital income. However, applying Corollary $2(i)$, we conclude that indeterminacy cannot occur in this case since Assumption 5.2 is not satisfied when $\alpha_{K K}<0$.

We conclude that distortions on capital or output market per se (see also the case of consumption externalities discussed below) do not seem to be empirically plausible sources of equilibrium indeterminacy.

\subsubsection{Distortions that only affect effective consumption}

In these examples distortions do neither affect the offer curve nor the real interest rate. Therefore, by Corollaries 1 and 2, indeterminacy always requires Assumptions 5.1 and 5.2.

With labor income taxation, both assumptions are only satisfied if $\phi_{L}<0$, implying that indeterminacy does not occur when tax rates are constant or vary positively with the tax base. For $\phi_{L}<0$, as we obtain configuration $(i)$ (see Table 2), indeterminacy occurs for $\sigma>\sigma_{H 1}=$ $\frac{-\phi_{L} s z_{L}+\left(1-z_{L}\right)[s-\theta(1-s)]}{\left(-\phi_{L} z_{L}\right)}$, provided that the elasticity of labor supply is high enough (see Table 1). ${ }^{26}$ For instance, when $\phi_{L}=-1^{27}$ and $z_{L}<0.5$ so that Assumption 1 (See Table 2) is verified, indeterminacy can emerge with $\sigma \geq 1$ for $z_{L}>[s-\theta(1-s)] /[1-\theta(1-s)] \equiv z_{l}^{*}=0.33$ under our calibration. This range of values for $z_{L}$ is in accordance with estimated average labor income tax rates for several European countries, as obtained in Mendoza et al. (1994) and in Volkerink et al (2002).

Using the equivalence result of Proposition 7 between labor income taxation and consumption externalities, we see that in this last case Assumption 1 implies $\chi<1$ and indeterminacy only occurs for $\chi>0$. Hence, indeterminacy is only possible when consumption externalities are of the "keepingup with the Joneses" type, occurring for $\sigma>\sigma_{H 1}=[s(1+\chi)-\theta(1-s)] / \chi$. Under our calibration, indeterminacy with a Cobb-Douglas technology emerges for $1>\chi>[s-\theta(1-s)] /(1-s)=0.5037$, which seems to be an excessive value. Indeed, although empirical values for these type of externalities do not seem to exist, Maurer and Meier (2008) found significant peer effects within several different groups of individuals, but in any case lower than 0.44. However, even if consumption externalities in our setup do not lead to indeterminacy under empirically relevant situations, we should notice that the indeterminacy mechanism involved is important since it is equivalent to

\footnotetext{
${ }^{26}$ Note that in this example, as in all the following ones, $\alpha_{\Gamma L}^{H}=\alpha_{\Gamma L}^{*}$. Therefore $\sigma_{H_{2}}$ does not exist (see Appendix 6.4, with $\alpha_{K K}=0$ ) so that footnote 16 applies.

${ }^{27}$ This corresponds to the case of a constant real government spending considered in Schmitt-Grohé and Uribe (1997) for a Ramsey model, and in Pintus (2003) and Gokan (2005) for a Woodford model.
} 
labor income taxation, a distortion that may lead to indeterminacy under relevant parameterizations, as shown above.

We will use this equivalence result to show that taxes may be used as stabilizers, i.e., we show below that labor income tax rates, that vary positively with the tax base, are able to eliminate indeterminacy due to the presence of positive consumption externalities, insulating therefore the economy from belief driven fluctuations. To see this consider a model where we have simultaneously consumption externalities with $\chi>0$ and labor taxation. In this case we obtain $\alpha_{j, i}=\beta_{j, i}=0$ for $i=K, L$ and $j=K, \Gamma, \alpha_{L, L}=$ $-\phi_{L} \frac{z_{L}}{1-z_{L}}+\chi\left(1-\phi_{L} \frac{z_{L}}{1-z_{L}}\right), \alpha_{L, K}=0$ and $\beta_{L, L}=-\alpha_{L, L} s=-\beta_{L, K}$. It is easy to see that by choosing the appropriate tax parameters, i.e. by choosing $\phi$ and $z_{L}$ such that $\phi_{L} \frac{z_{L}}{1-z_{L}}=\frac{\chi}{1+\chi}$, the government is able to set at zero all the distortion parameters, i.e., it is able to recover the perfect competition framework where indeterminacy, and therefore cycles driven by self-fulfilling volatile expectations, do not emerge. For example for $\chi=0.52$, as referred above, indeterminacy would emerge in the Cobb-Douglas case when there is no taxation. However, choosing combinations of $\phi_{L}$ and $z_{L}$ such that $\phi_{L} \frac{z_{L}}{1-z_{L}}=0.342$, (i.e. for example $\phi_{L}=1$ and $z_{L}=0.255, \phi_{L}=1.145$ and $z_{L}=0.23$ or $\phi_{L}=0.453$ and $z_{L}=0.43$, all of them empirically plausible) the economy behaves as an economy without distortions and indeterminacy does not emerge.

\subsubsection{Distortions that affect the generalized offer curve but not the real interest rate}

Since in these examples distortions do not affect the real interest rate, by Corollary 2 (iii), indeterminacy always requires Assumption 5.2. ${ }^{28}$

With leisure externalities, where only the parameter $\alpha_{\Gamma L}$ is affected, Assumption 5.2 implies that $\mu=\alpha_{\Gamma L}<0$, which also ensures Assumption 5.1. Note also that, since $\mu>-1$ under Assumption 4 and $\varepsilon_{\gamma} \geq 1$, we have $0<\varepsilon_{\Gamma L}=\varepsilon_{\gamma}+\mu<1$, i.e. the generalized offer curve is still positively sloped (See (6)). In this example $\sigma_{H_{1}}=\frac{s-\theta(1-s)(1+\mu)}{-\mu}$ so that $\sigma>\sigma_{H_{1}} \Leftrightarrow$ $\mu<\mu^{1} \equiv-[s-\theta(1-s)] /[1-\theta(1-s)]$, where $\alpha_{\Gamma L}^{b}<\mu^{1}<0$, covering some parameterizations of configurations $(i)$ and $(i i)$. See Table 2. Hence, from Proposition 5, and assuming for simplicity that $\varepsilon_{\gamma}=1$, indeterminacy may emerge with $\sigma=1$ when $\mu<\mu^{1}$. Under our calibration, we obtain $\mu^{1} \simeq-0.33$, a value that does not seem to be exaggerated. Even though we

\footnotetext{
${ }^{28}$ Note that in these examples $\alpha_{\Gamma L}^{H}=\alpha_{\Gamma L}^{*}$, so that $\sigma_{H_{2}}=+\infty$. See Appendix 6.4, with $\alpha_{K K}=0$. In this case, $\sigma_{H_{2}}>\sigma_{F}$ and Assumption 9 is verified. Also, since $\sigma_{H_{2}} \in$ $\left(\frac{\left(s-\beta_{L L}\right)}{\left(1+\alpha_{L L}\right)},+\infty\right)$ does not exist, footnote 16 of Proposition 5 applies.
} 
do not have empirical estimates for the degree of leisure externalities, note that this value is significantly closer to zero than the values used in related literature, as for instance the value $\mu=-1.23$ considered in Benhabib and Farmer (2000).

Using the equivalence result of Proposition 8 between leisure externalities and unemployment insurance with efficiency wages, we see that in this last case indeterminacy occurs with $\sigma=1$ if $\frac{-z}{z+(1-z) n}<-0.33$. We can see that for empirically plausible values of $n$ and $z$ indeterminacy occurs. For instance, if the employment rate is $n=0.95$, indeterminacy occurs as soon as the replacement ratio $z$ is higher than 0.32 , covering the case of most developed economies.

In the example with unemployment benefits and unions, based on Dufourt et al. (2008), Assumption 5.2 required for indeterminacy is always satisfied, and so it is Assumption 5.1. One can check that $\sigma_{H 1}=s<1$ and that $\sigma_{F}=1-\frac{2(1-s)(1-\theta / 2)(1-\alpha s)}{2(1-2 s)+2 s \alpha}<1$. Since $\epsilon_{\gamma}=1$, indeterminacy prevails when $\sigma=1$, independently of the degree of union power. This result shows that the existence of financed constrained workers together with unemployment benefits, a situation characterizing many developed economies, is likely to create indeterminacy and also complex employment fluctuations through the occurrence of Hopf and flip bifurcations.

We conclude that with plausible labor market imperfections, either leisure externalities, unemployment benefits or labor income taxation, indeterminacy emerges under reasonable degrees of capital-labor substitution.

\section{Concluding Remarks}

With our general analysis of the role of market distortions on local dynamics and the different examples of specific distortions presented above, we were able to emphasize several interesting results, some of them already latent in previous works, but which are here confirmed, generalized and highlighted. First, our work enabled us to find classes of specific distortions within which equivalence results are obtained. Specific distortions belonging to the same class are observational equivalent and have the same consequences in terms of the local dynamic behavior of (cyclical) aggregate capital and labor. Second, capital market distortions per se do not seem to play a major role for the occurrence of indeterminacy. On the contrary, bifurcations and indeterminacy emerge under labor market rigidities, without imposing strange or implausible restrictions, whereas for output market distortions, indeterminacy requires conditions that might be considered less relevant from an empirical point of view. These findings suggest that the functioning of la- 
bor markets, which in the real world show significant deviations from the competitive paradigm, may be responsible for the persistency along business fluctuations and for the existence of expectation driven cycles. ${ }^{29}$ Further analysis on this issue is therefore important for future research.

A possible explanation for these results may be linked to the fact that future expectations, which open the room for fluctuations driven by selffulfilling expectations, only affect the current decisions of consumers/workers, rendering distortions that affect the intertemporal trade-off of consumers/workers more important than those affecting the capital accumulation equation. ${ }^{30}$ Strategic considerations by firms owning productive capital, which are usually disregarded, may render future expectations of capitalists/producers relevant, and change the results. Although some works have already considered some of these aspects ${ }^{31}$ further research on this issue is welcome.

\section{Appendix}

\subsection{Existence of a steady state}

Proposition 9 (Existence of the normalized steady state) $\left(K^{*}, L^{*}\right)=$ $(1,1)$ is a stationary solution of the dynamic system (4)-(5) if and only if $A=\theta /\left(\beta \varrho(1,1)>0\right.$ and $B=[\beta \varrho(1,1) \Gamma(1,1)]^{-1} \theta \Omega(1,1)>0$.

Proof. A stationary equilibrium of the dynamic system (4)-(5) is a solution $(K, L)=\left(K_{t-1}, L_{t}\right)$ for all $t$, that satisfies $A \varrho(K, L)=\theta / \beta$ and $(A / B) \Omega(K, L) L=\Gamma(K, L)$. The existence of a steady state can be established by choosing appropriately the two scaling parameters $A>0$ and $B>0$ so as to ensure that one steady state coincides with $(K, L)=(1,1)$. From the first equation, we obtain a unique solution $A=\theta /(\beta \varrho(1,1)>0$. Substituting this into the second equation we then obtain the unique solution for $B=[\beta \varrho(1,1) \Gamma(1,1)]^{-1} \theta \Omega(1,1)>0$.

\footnotetext{
${ }^{29}$ Dufourt et al. (2007) replicate the fluctuations and persistence of unemployment data, considering i.i.d. sunspot shocks on expectations in a model with unions and unemployment benefits. Chari et al. (2007) show quantitatively that labor market frictions constitute one of the most promising mechanisms through which shocks on fundamentals lead to business fluctuations.

${ }^{30}$ Indeed, in all the usual macrodynamic frameworks, including the Ramsey and overlapping generations models, firms just rent productive capital, accummulated from past savings of consumers/capitalists, so that future expectations do not directly influence capital accumulation.

${ }^{31}$ See for instance d'Aspremont et. al (2000).
} 


\subsection{Local Dynamics - Proof of Proposition 5}

Our proof of Proposition 5 is based on the geometrical method developed in Grandmont et al. (1998). We consider that $s$ and $\theta$ are fixed throughout the analysis and, for given different values of $\alpha_{i j}$ and $\beta_{i j}$ satisfying all the Assumptions considered, we study how $(T, D)$ change as $\varepsilon_{\gamma}$ and $\sigma$ vary in their admissible ranges.

The half-line $\Delta$ We start by discussing how $T$ and $D$ move in the space $(T, D)$ as $\varepsilon_{\gamma}$ is made to continuously change. From (8) and (9), the locus of points $(T, D)=\left(T\left(\varepsilon_{\gamma}\right), D\left(\varepsilon_{\gamma}\right)\right)$ as $\varepsilon_{\gamma}$ varies in $[1,+\infty)$ describes a halfline $\Delta$ in the plane $(T, D)$, starting for $\varepsilon_{\gamma}=1$ at $(T(1), D(1))=\left(T_{1}, D_{1}\right)$ and pointing upwards as $\varepsilon_{\gamma}$ increases to $+\infty$ (since $D$ increases with $\varepsilon_{\gamma}$, see Lemma 1), with a slope $S$ equal to:

$$
S=1+\theta \alpha_{K, K}-\theta \frac{1-s-\beta_{K, K}}{\sigma}
$$

Note that the half-line $\Delta$ shifts when $\sigma$ changes, because its slope $S$ and its initial point $\left(T_{1}, D_{1}\right)$ depend on $\sigma$.

The next Lemma, that can be easily proved using (10), Assumption 1 and simple analytical computations, will help us understanding why results are different according to whether $\alpha_{K K} \leq 0$ or $\alpha_{K K}>0$.

Lemma 2 Under Assumption 1 and defining $\sigma_{T}$ as in (20), we have the following:

1. If $\alpha_{K K}<0$ then $0<S<1$ for all $\sigma \in\left(\frac{s-\beta_{L L}}{1+\alpha_{L L}},+\infty\right)$ and $\lim _{\sigma \rightarrow+\infty} S<1$

2. If $\alpha_{K K}=0$ then $0<S<1$ for all $\sigma \in\left(\frac{s-\beta_{L L}}{1+\alpha_{L L}},+\infty\right)$ and $\lim _{\sigma \rightarrow+\infty} S=1$

3. If $\alpha_{K K}>0$, then:

(a) $1<S<1+\theta \alpha_{K, K}$ if and only if $\sigma \in\left(\sigma_{T},+\infty\right)$, and $\lim _{\sigma \rightarrow+\infty} S>1$

(b) $S=1$ if and only if $\sigma=\sigma_{T}$

(c) $0<S<1$ if and only if $\sigma \in\left(\frac{s-\beta_{L L}}{1+\alpha_{L L}}, \sigma_{T}\right)$ 
The half-line $\Delta_{1}$ Let us now discuss the behavior of $\left(T_{1}, D_{1}\right)$ as $\sigma$ varies. From (8) and (9), the locus of points $\left(T_{1}(\sigma), D_{1}(\sigma)\right)$ obtained as $\sigma$ decreases from $+\infty$ to $\left(s-\beta_{L L}\right) /\left(1+\alpha_{L L}\right)$ describes a half-line $\Delta_{1}$, starting for $\sigma=+\infty$ at $\left(T_{1}(+\infty), D_{1}(+\infty)\right)=\left(1+\frac{\left(1+\theta \alpha_{K K}\right)\left(1+\alpha_{\Gamma L}\right)-\theta \alpha_{\Gamma K} \alpha_{K L}}{1+\alpha_{L L}}, \frac{\left(1+\theta \alpha_{K K}\right)\left(1+\alpha_{\Gamma L}\right)-\theta \alpha_{\Gamma K} \alpha_{K L}}{1+\alpha_{L L}}\right)$, and pointing upwards as $\sigma$ decreases (since $D_{1}$ is decreasing in $\sigma$, see Lemma 1). The slope of the half-line $\Delta_{1}, S_{1} \equiv \frac{d D_{1} / d \sigma}{d T_{1} / d \sigma}$, is given by:

$$
S_{1}=\frac{\left(\alpha_{\Gamma L}^{* *}-\alpha_{\Gamma L}\right)\left[\left(s-\beta_{L, L}\right)\left(1+\theta \alpha_{K K}\right)-\theta\left(1+\alpha_{L, L}\right)\left(1-s-\beta_{K, K}\right)\right]}{\left(\alpha_{\Gamma L}^{a}-\alpha_{\Gamma L}\right)\left(s-\beta_{L L}\right)\left(1+\theta \alpha_{K K}\right)}
$$

with $\alpha_{\Gamma L}^{* *}$ and $\alpha_{\Gamma L}^{a}$ defined respectively in Assumption 4 and in (12) of Appendix 6.3.1.

The next Lemma will help us understanding why results may be different in the three different configurations considered for $\alpha_{\Gamma L}$.

Lemma 3 Under Assumptions 1-6, defining $\alpha_{\Gamma L}^{b}$ and $\alpha_{\Gamma L}^{c}$ as in Appendix 6.3.1, and $S_{B} \in(-1,0)$ as in (17) in Appendix 6.3.2, the following holds:

(i) For $\alpha_{\Gamma L}>\alpha_{\Gamma L}^{b},\left|S_{1}\right|>1$;

(ii) For $\alpha_{\Gamma L} \in\left(\alpha_{\Gamma L}^{c}, \alpha_{\Gamma L}^{b}\right), S_{1} \in\left(-1, S_{B}\right)$, where $S_{B}$ is the slope of $\Delta_{1}$ when $\Delta_{1}$ goes through point $B$;

(iii) For $\alpha_{\Gamma L}<\alpha_{\Gamma L}^{c}, S_{1} \in\left(S_{B}, 0\right)$.

Proof : First note that, given the definitions of the critical values of $\alpha_{\Gamma L}$ in Appendix 6.3.1, $S_{1}$ can be written as in (11) but also in several different ways (see (14)-(16)). Under Assumption 4, $\alpha_{\Gamma L}>\alpha_{\Gamma L}^{* *}$ and the numerator of $S_{1}$ in (11) is negative, given Assumption 1. Hence, under Assumption 1, $S_{1}<0$ iff $\alpha_{\Gamma L}<\alpha_{\Gamma L}^{a}$ and $S_{1}>0$ iff $\alpha_{\Gamma L}>\alpha_{\Gamma L}^{a}$. Using (14) we can see that, given Assumption 5.2, $S_{1} \notin(0,1)$ and we obtain $S_{1}>1$ for $\alpha_{\Gamma L} \geq \alpha_{\Gamma L}^{a}$. Also, using (15), we see that, under Assumption 1, $S_{1}<-1$ for $\alpha_{\Gamma L}^{a}>\alpha_{\Gamma L}>\alpha_{\Gamma L}^{b}$. This proves Lemma 3. $(i)$. Note also that, using (11) and Assumption 1, $S_{1}$ is decreasing in $\alpha_{\Gamma L}$ for $\alpha_{\Gamma L}^{a}>\alpha_{\Gamma L}>\alpha_{\Gamma L}^{* *}$. Hence, by decreasing $\alpha_{\Gamma L}$ further from $\alpha_{\Gamma L}^{b}$ until $\alpha_{\Gamma L}^{* *}, S_{1}$ must increase from -1 until 0 (see (15) and (11)), and $S_{1}$ cannot cross the value $S_{B} \in(-1,0)$ twice for $\alpha_{\Gamma L}<\alpha_{\Gamma L}^{b}$. This, by (16), implies that only one of the roots $\alpha_{\Gamma L}^{c}, \alpha_{\Gamma L}^{c+}$ can lay in the interval $\left(\alpha_{\Gamma L}^{* *}, \alpha_{\Gamma L}^{b}\right)$. We now show that this root must be $\alpha_{\Gamma L}^{c}$. Indeed, using (16) and (15), and since $S_{B} \in(-1,0)$ under the Assumptions of Lemma 3 (see (17)), we have that the inequality $\left(\alpha_{\Gamma L}^{b}-\alpha_{\Gamma L}\right)\left[2\left(s-\beta_{L, L}\right)\left(1+\theta \alpha_{K K}\right)-\theta\left(1+\alpha_{L, L}\right)\left(1-s-\beta_{K, K}\right)\right]>$ 
$\frac{\left(\alpha_{\Gamma L}-\alpha_{\Gamma L}^{c}\right)\left(\alpha_{\Gamma L}-\alpha_{\Gamma L}^{c+}\right)\left(1+\alpha_{L, L}\right)\left(1-s-\beta_{K, K}\right) \theta\left(1+\theta \alpha_{K, K}\right)}{\left[2\left(1+\alpha_{L, L}\right)+\left(1+\theta \alpha_{K K}\right)\left(\alpha_{\Gamma L}-\alpha_{\Gamma L}^{L}\right)\right]}$ is satisfied for any value of $\alpha_{\Gamma L}<$ $\alpha_{\Gamma L}^{a}$, and in particular for $\alpha_{\Gamma L}=\alpha_{\Gamma L}^{b}$. (Note that $\alpha_{\Gamma L}^{b}<\alpha_{\Gamma L}^{a}$, since $S_{1}=$ $-1<0$ for $\alpha_{\Gamma L}=\alpha_{\Gamma L}^{b}$ and $S_{1}<0$ iff $\left.\alpha_{\Gamma L}<\alpha_{\Gamma L}^{a}\right)$. Therefore, using the last expression with $\alpha_{\Gamma L}=\alpha_{\Gamma L}^{b}$, together with Assumptions 1 and 6, we have $\left(\alpha_{\Gamma L}^{b}-\alpha_{\Gamma L}^{c}\right)\left(\alpha_{\Gamma L}^{b}-\alpha_{\Gamma L}^{c+}\right)<0$, and the following is satisfied: $\alpha_{\Gamma L}^{c}<$ $\alpha_{\Gamma L}^{b}<\alpha_{\Gamma L}^{c+}$. Hence, $S_{1}$ crosses $S_{B}$ for $\alpha_{\Gamma L}=\alpha_{\Gamma L}^{c}$ and $-1<S_{1}<S_{B}$ for $\alpha_{\Gamma L}^{b}>\alpha_{\Gamma L}>\alpha_{\Gamma L}^{c}$, while $0>S_{1}>S_{B}$ for $\alpha_{\Gamma L}<\alpha_{\Gamma L}^{c}$. This proves Lemma 3. $(i i)$ and $(i i i)$.

We now characterize the local stability properties of the steady state for each configuration defined in Lemma 3. We use geometrical arguments whenever possible, by referring to Figures 1-3. For any given configuration, remember that the half-line $\Delta$, which starts for $\varepsilon_{\gamma}=1$ on half-line $\Delta_{1}$, points upwards to the right as $\varepsilon_{\gamma}$ increases from 1 to $+\infty$. Also, as $\sigma$ decreases from $+\infty$ to $\left(s-\beta_{L L}\right) /\left(1+\alpha_{L L}\right)$, the half line $\Delta$ becomes less steep (see (10)) and its initial point $\left(T_{1}, D_{1}\right)$ moves upwards along the half line $\Delta_{1}\left(D_{1}\right.$ is decreasing in $\sigma$, Lemma 1 ). Recall also that the half-line $\Delta_{1}$ always start for $\sigma=+\infty$ on line $(A C)$ between points $A$ and $C$, due to Assumptions 3, 5.1 and 6 . Finally remember that the half-line $\Delta_{1}$ points upwards as $\sigma$ decreases from $+\infty$ to $\left(s-\beta_{L L}\right) /\left(1+\alpha_{L L}\right)$. The critical values of $\alpha_{\Gamma L}, \sigma$ and $\varepsilon_{\gamma}$ used below are given in Section 6.3.

Configuration $(i)\left(\alpha_{\Gamma L}>\alpha_{\Gamma L}^{b}\right)$ Using Lemma 3, we have $\left|S_{1}\right|>1$. Therefore, the half-line $\Delta_{1}$ that starts on the line $(A C)$, between $A$ and $C$, points upwards with a slope $S_{1}$ strictly greater than 1 or strictly smaller than -1 , crossing neither $(A B)$, nor $(A C)$ (see Figure 1). However, since $\Delta_{1}$ crosses the segment $[B C]$, there is a critical value for $\sigma, \sigma_{H_{1}}>\frac{s-\beta_{L L}}{1+\alpha_{L L}}$ such that $D_{1}\left(\sigma_{H_{1}}\right)=1$ (see (18)). If $\sigma \leq \sigma_{H_{1}}$, the half-line $\Delta$ starts on $\Delta_{1}$ above $[B C]$ and since it points upwards to the right, it crosses the line $(A C)$, above point $C$, for $\varepsilon_{\gamma}=\varepsilon_{\gamma_{T}}$. Accordingly, the steady state is a source for $1 \leq \varepsilon_{\gamma}<\varepsilon_{\gamma_{T}}$, undergoes a transcritical bifurcation for $\varepsilon_{\gamma}=\varepsilon_{\gamma_{T}}$ and becomes a saddle for $\varepsilon_{\gamma}>\varepsilon_{\gamma_{T}}$. If $\sigma>\sigma_{H_{1}},\left(T_{1}(\sigma), D_{1}(\sigma)\right)$ is inside the triangle $(A B C)$ and the half-line $\Delta$ must also cross the line $(B C)$, but Hopf bifurcations only occur if the crossing point is on the left of point $C$, so that the half-line $\Delta$ crosses $[B C]$ in its interior. We can see geometrically that, by continuity, for $\sigma$ higher but close to $\sigma_{H_{1}}$ the half-line $\Delta$ crosses the segment $[B C]$ in its interior. However, for higher values of $\sigma>\sigma_{H_{1}}$ this may not happen. Let us define $\sigma_{H_{2}} \in\left(\sigma_{H_{1}},+\infty\right)$ as a critical value for $\sigma$ such that the half line $\Delta$ goes through point $C$. 
Consider first that $\alpha_{K K}<0$, which means that $S<1$ and that $\lim _{\sigma \rightarrow+\infty} S<$ 1 (see Lemma 2). In Appendix 6.4 we show that $\sigma_{H_{2}}$ is unique and for $\sigma>\sigma_{H_{2}}$ the half-line $\Delta$ crosses first $(A C)$ below point $C$, crossing $(B C)$ on the right of point $C$ so that no Hopf bifurcations occur, while the reverse occurs for $\sigma_{H_{1}}<\sigma<\sigma_{H_{2}}$.

When $\alpha_{K K}=0$, we still have $S<1$, although $\lim _{\sigma \rightarrow+\infty} S=1$. Due to this, $\sigma_{H_{2}}$ may not exist. As shown in Appendix 6.4, this happens if $\alpha_{\Gamma L}^{H} \leq \alpha_{\Gamma L}^{*}$, implying that the half-line $\Delta$ crosses the segment $[B C]$ in its interior and then crosses $(A C)$ above point $C$, for all $\sigma \in\left(\sigma_{H_{1}},+\infty\right)$. Otherwise, if $\alpha_{\Gamma L}^{H}>\alpha_{\Gamma L}^{*}$ then $\sigma_{H_{2}}$ exists and everything is similar to the case of $\alpha_{K K}<0$.

Assuming now that $\alpha_{K K}>0$, there exists the critical value $\sigma_{T}>(s-$ $\left.\beta_{L L}\right) /\left(1+\alpha_{L L}\right)$ for $\sigma$ such that $S\left(\sigma_{T}\right)=1$ and $S>1$ for $\sigma>\sigma_{T}$ (see Lemma $2)$. To simplify the exposition we assume that:

Assumption 7 If $\alpha_{K K}>0$, then $\sigma_{T}>\max \left\{\sigma_{H_{1}}, \sigma_{F}\right\}$.

where $\sigma_{F}$ is introduced for further reference and is such that the half-line $\Delta_{1}$ crosses line $(A B)$, i.e., $1+D_{1}\left(\sigma_{F}\right)+T_{1}\left(\sigma_{F}\right)=0$ (see (19).

This Assumption implies that for $\sigma \geq \sigma_{T}$ the half-line $\Delta$ starts within the triangle $(A B C)$ and points upwards with a slope higher than 1 . Hence, when $\sigma \geq \sigma_{T}$, the half-line $\Delta$ only crosses $[B C]$ on the left of point $C$. For $\sigma_{T}>\sigma>\sigma_{H_{1}}$ we further assume that:

Assumption 8 If $\alpha_{K K}>0$ and $\sigma_{T}>\sigma>\max \left\{\sigma_{H_{1}}, \sigma_{F}\right\}$, then $\varepsilon_{\gamma_{H}}<\varepsilon_{\gamma_{T}}$.

This Assumption means that $\sigma_{H_{2}} \in\left(\sigma_{H_{1}},+\infty\right)$ does not exist and is ensured if (24) of Appendix 6.4 is satisfied. It implies that, when $\sigma_{H_{1}}<\sigma<\sigma_{T}$, the half-line $\Delta$ crosses first the segment $[B C]$ and then line $(A C)$ above $C$.

Configuration (ii) $\left(\alpha_{\Gamma L} \in\left(\alpha_{\Gamma L}^{c}, \alpha_{\Gamma L}^{b}\right)\right) \quad$ Using Lemma 3, we have that $S_{1} \in\left(-1, S_{B}\right)$. Therefore, in this configuration, the half-line $\Delta_{1}$ points upwards to the left, crossing line $(A B)$ above point $B$ for $\sigma=\sigma_{F}$ (see Figure 2).

Consider first that $\alpha_{K K} \leq 0$ so that $S<1$ (see Lemma 2). When $\sigma<\sigma_{F}$, $\left(T_{1}(\sigma), D_{1}(\sigma)\right)$ is below line $(A B)$ and above B. Since the half-line $\Delta$ points upwards it does not cross $[B C]$, but crosses $(A B)$ before crossing $(A C)$. When $\sigma_{F} \leq \sigma \leq \sigma_{H_{1}},\left(T_{1}(\sigma), D_{1}(\sigma)\right)$ is above (or over) $(A B)$ and $[B C]$. Then, the half-line $\Delta$ only crosses line $(A C)$. As in the previous configuration, when $\sigma>\sigma_{H_{1}}$ the point $\left(T_{1}(\sigma), D_{1}(\sigma)\right)$ is inside the triangle $(A B C)$ and, for $\alpha_{K K}<0$, there is a unique value $\sigma_{H_{2}}$ such that for $\sigma_{H_{1}}<\sigma<\sigma_{H_{2}}$, the half-line $\Delta$ crosses first $[B C]$ and then line $(A C)$ above $C$, and for $\sigma>\sigma_{H_{2}}$, 
the half-line $\Delta$ only crosses line $(A C)$ below $C$ (see Appendix 6.4). Also, when $\alpha_{K K}=0$, if $\sigma_{H_{2}}$ does not exist, for $\sigma>\sigma_{H_{1}}$ the half-line $\Delta$ crosses first $[B C]$ and then line $(A C)$ above $C$. If $\sigma_{H_{2}}$ exists then everything is similar to the case where $\alpha_{K K}<0$.

Consider now that $\alpha_{K K}>0$. In this case, the critical value $\sigma_{T}>0$ exists and, as in the previous configuration, we consider Assumption 7 so that $\sigma_{T}>\sigma_{H_{1}}>\sigma_{F}$. Therefore, for $\sigma<\sigma_{H_{1}}, S$ is still smaller than 1 and we obtain the same results as before. When $\sigma_{H_{1}}<\sigma<\sigma_{T},\left(T_{1}(\sigma), D_{1}(\sigma)\right)$ is inside the triangle $(A B C)$ and, under Assumption 8, the half-line $\Delta$ crosses first $[B C]$, and then $(A C)$ above point $C$. When $\sigma \geq \sigma_{T}, S$ becomes greater than 1 , which means that $\Delta$ only crosses $[B C]$.

Configuration (iii) $\left(\alpha_{\Gamma L}<\alpha_{\Gamma L}^{c}\right) \quad$ Using Lemma 3, we have that $S_{1} \in$ $\left(S_{B}, 0\right)$, with $S_{B} \in(-1,0)$. Therefore, the slope $S_{1}$ is negative and greater than -1 , and the half line $\Delta_{1}$, that points upwards to the left, crosses line $(A B)$ below point $B$ (see Figure 3 ). In this configuration, a new critical value, $\sigma_{H_{3}}$, the value of $\sigma$ such that the half line $\Delta$ goes through point $B$, becomes relevant. In Appendix 6.5 we prove that in this configuration, there exists a unique critical value $\sigma_{H_{3}} \in\left(\sigma_{H_{1}}, \sigma_{F}\right)$ such that the half-line $\Delta$ goes through point $B$ and crosses $[B C]$ on the right of $B$ for $\sigma>\sigma_{H_{3}}$.

We begin by assuming $\alpha_{K, K} \leq 0$, so that $S<1$. Recall now that when $\sigma_{H_{2}}>\frac{s-\beta_{L L}}{1+\alpha_{L L}}$ exists (its existence is always ensured when $\alpha_{K, K}<0$, see Appendix 6.4) the half-line $\Delta$ crosses $(B C)$ on the left of $C$ for $\sigma<\sigma_{H_{2}}$ and on the right of $C$ for $\sigma>\sigma_{H_{2}}$. We can now see, geometrically, that although in Configuration (ii) $\sigma_{H_{2}}>\sigma_{F}$, in the current configuration if $\sigma_{H_{2}}$ exists it may be higher or lower than $\sigma_{F}$. To simplify the exposition we consider the following Assumption:

Assumption 9 If $\alpha_{\Gamma L}<\alpha_{\Gamma L}^{c}, \alpha_{K, K} \leq 0$ and $\sigma_{H_{2}}>\frac{s-\beta_{L L}}{1+\alpha_{L L}}$, then $\sigma_{H_{2}}>\sigma_{F}$.

Hence, for $\sigma<\sigma_{H_{3}}, \Delta$ starts on the left-side of line $(A B)$, crosses line $(A B)$ above $B$ and then crosses line $(A C)$. For $\sigma_{H_{3}}<\sigma<\sigma_{F}, \Delta$ also starts on the left-side of $(A B)$, but crosses $(A B)$ below $B$, the segment $[B C]$, and $(A C)$ above $C$. Then, for $\sigma_{F} \leq \sigma<\sigma_{H_{2}},\left(T_{1}(\sigma), D_{1}(\sigma)\right)$ is inside $(A B C)$, and $\Delta$ crosses $[B C]$ and $(A C)$ above $C$. For $\sigma>\sigma_{H_{2}},\left(T_{1}(\sigma), D_{1}(\sigma)\right)$ is still inside $(A B C)$ and $\Delta$ crosses $(A C)$ below $C$. This last case does not appear if $\sigma_{H_{2}}$ does not exist. See Appendix 6.4, for $\alpha_{K, K}=0$.

We consider now the case where $\alpha_{K, K}>0$. Under Assumptions 7 and 8, $\sigma_{T}>\sigma_{F}\left(>\sigma_{H_{3}}\right)$. Then, for $\sigma<\sigma_{H_{3}}$, the half-line $\Delta$ crosses first line $(A B)$ above $B$ and then it crosses line $(A C)$. For $\sigma_{H_{3}}<\sigma<\sigma_{F}, \Delta$ crosses $(A B)$ below $B$, the segment $[B C]$ and, under Assumption 8, it crosses line $(A C)$ 
above $C$. For $\sigma_{F} \leq \sigma<\sigma_{T}, \Delta$ starts inside $(A B C)$ with a slope smaller than 1. Then, it crosses $[B C]$ and, given again 8 , it crosses line $(A C)$ above $C$. For $\sigma \geq \sigma_{T}$, the slope $S$ being greater than $1, \Delta$ only crosses $[B C]$.

All these results are summarized in Table 1.

\subsection{Critical values of the parameters}

\subsubsection{Definitions and expressions for critical values of $\alpha_{\Gamma L}$}

$\alpha_{\Gamma L}^{*}$ is such that $S_{1}=1$ and is given in Assumption 5.2.

$\alpha_{\Gamma L}^{H}$ is such that $D_{1}(+\infty)=1$ and is given in Assumption 5.1.

$\alpha_{\Gamma L}^{L}$ is such that $D_{1}(+\infty)=-1$ and is given in Assumption 6 .

$\alpha_{\Gamma L}^{* *}$ is such that $S_{1}\left(\alpha_{\Gamma L}^{* *}\right)=0$ and is given in Assumption 4.

$\alpha_{\Gamma L}^{a}$ is such that $S_{1}\left(\alpha_{\Gamma L}^{a}\right)=\infty$ and is given by:

$$
\begin{aligned}
\alpha_{\Gamma L}^{a} \equiv & \left\{\theta ( 1 + \alpha _ { L , L } ) \left[\left(1+\alpha_{L, L}\right)\left(1-s-\beta_{K, K}\right)+\alpha_{L, K}\left(1-s+\beta_{K, L}\right)\right.\right. \\
& \left.+\alpha_{K L} \frac{\left(1-s-\beta_{K, K}\right)\left(s-\beta_{L L}\right)}{\left(1-s+\beta_{K L}\right)}\right]-\left(s-\beta_{L L}\right)\left(1-\theta \alpha_{K, K} \alpha_{L, L}-\theta \alpha_{\Gamma, K} \alpha_{K, L}\right) \\
& \left.-\left(1+\alpha_{L, L}\right) \beta_{\Gamma, L}\right\} /\left(s-\beta_{L L}\right)\left(1+\theta \alpha_{K K}\right)
\end{aligned}
$$

$\alpha_{\Gamma L}^{b}$ is such that $S_{1}\left(\alpha_{\Gamma L}^{b}\right)=-1$ and, using (12), is given by:

$\alpha_{\Gamma L}^{b}=\frac{\alpha_{\Gamma L}^{a}\left(s-\beta_{L L}\right)\left(1+\theta \alpha_{K K}\right)+\left[\left(s-\beta_{L L}\right)\left(1+\theta \alpha_{K K}\right)-\theta\left(1+\alpha_{L, L}\right)\left(1-s-\beta_{K, K}\right)\right] \alpha_{\Gamma L}^{* *}}{2\left(s-\beta_{L, L}\right)\left(1+\theta \alpha_{K K}\right)-\theta\left(1+\alpha_{L, L}\right)\left(1-s-\beta_{K, K}\right)}$

$\alpha_{\Gamma L}^{c}$ is the lower root of equation $S_{1}=S_{B}$ (see (16)), where $S_{B}$ is given in (17). The other root is noted $\alpha_{\Gamma L}^{c+}$.

\subsubsection{Expressions for $S_{1}$ in terms of critical values of $\alpha_{\Gamma L}$}

$S_{1}$, given in (11), can also be written as:

$$
\begin{aligned}
S_{1} & =1+\frac{\left(\alpha_{\Gamma L}-\alpha_{\Gamma L}^{*}\right) \theta\left(1+\alpha_{L L}\right)\left(1-s-\beta_{K K}\right)}{\left(\alpha_{\Gamma L}^{a}-\alpha_{\Gamma L}\right)\left(s-\beta_{L L}\right)\left(1+\theta \alpha_{K K}\right)} ; \\
S_{1} & =-1+\frac{\left(\alpha_{\Gamma L}^{b}-\alpha_{\Gamma L}\right)\left[2\left(s-\beta_{L, L}\right)\left(1+\theta \alpha_{K K}\right)-\theta\left(1+\alpha_{L, L}\right)\left(1-s-\beta_{K, C_{(}}\right)\right]}{\left(\alpha_{\Gamma L}^{a}-\alpha_{\Gamma L}\right)\left(s-\beta_{L L}\right)\left(1+\theta \alpha_{K K}\right)} \\
S_{1} & =S_{B}+\frac{\left(\alpha_{\Gamma L}-\alpha_{\Gamma L}^{c}\right)\left(\alpha_{\Gamma L}-\alpha_{\Gamma L}^{c+}\right) \theta\left(1+\alpha_{L, L}\right)\left(1-s-\beta_{K, K}\right)}{\left(\alpha_{\Gamma L}^{a}-\alpha_{\Gamma L}\right)\left(s-\beta_{L L}\right)\left[2\left(1+\alpha_{L, L}\right)+\left(1+\theta \alpha_{K, K}\right)\left(\alpha_{\Gamma, L}-\alpha_{\Gamma, L}^{L}\right)\right]}(16)
\end{aligned}
$$

where $S_{B}$ is the slope of $\Delta_{1}$ when $\Delta_{1}$ goes through point $B=(-2,1)$, satisfying $S_{B} \in(-1,0)$ under Assumptions 1, 5.1 and 6, and being given by: 


$$
S_{B} \equiv \frac{-\left(1+\theta \alpha_{K K}\right)\left(\alpha_{\Gamma L}^{H}-\alpha_{\Gamma L}\right)}{2\left(1+\alpha_{L L}\right)+\left(1+\theta \alpha_{K K}\right)\left(\alpha_{\Gamma L}-\alpha_{\Gamma L}^{L}\right)} \in(-1,0)
$$

\subsubsection{Definitions and expressions for critical values of $\sigma$}

$\sigma_{H_{1}}$ is the critical value of $\sigma$ such that $D_{1}\left(\sigma_{H_{1}}\right)=1$ and is given by:

$\sigma_{H_{1}} \equiv \frac{s-\beta_{L, L}}{1+\alpha_{L, L}}+\frac{\left[\left(s-\beta_{L, L}\right)\left(1+\theta \alpha_{K K}\right)-\theta\left(1+\alpha_{L, L}\right)\left(1-s-\beta_{K, K}\right)\right]\left(\alpha_{\Gamma, L}-\alpha_{\Gamma, L}^{* *}\right)}{\left(1+\alpha_{L, L}\right)\left(1+\theta \alpha_{K K}\right)\left(\alpha_{\Gamma, L}^{H}-\alpha_{\Gamma, L}\right)}$.

$\sigma_{H_{2}}$ is a critical value of $\sigma$ such that the half-line $\Delta$ goes through the point $(T, D)=(2,1)$, i.e., goes through point $C .{ }^{32}$ Note that $\varepsilon_{\gamma_{T}}=\varepsilon_{\gamma_{H}}$ for $\sigma=\sigma_{H_{2}}$.

$\sigma_{H_{3}}$ is the critical value of $\sigma$ such that the half line $\Delta$ goes through the point $(T, D)=(-2,1)$, i.e., goes through point $B .^{33}$ Note that $\varepsilon_{\gamma_{F}}=\varepsilon_{\gamma_{H}}$ for $\sigma=\sigma_{H_{3}}$.

The critical value $\sigma_{F}$ is defined by $1+D_{1}\left(\sigma_{F}\right)+T_{1}\left(\sigma_{F}\right)=0$, $\sigma_{F} \equiv \frac{s-\beta_{L, L}}{1+\alpha_{L, L}}+\frac{\left[2\left(s-\beta_{L, L}\right)\left(1+\theta \alpha_{K K}\right)-\theta\left(1+\alpha_{L, L}\right)\left(1-s-\beta_{K, K}\right)\right]\left(\alpha_{\Gamma, L}^{b}-\alpha_{\Gamma, L}\right)}{2\left(1+\alpha_{L, L}\right)\left(1+\theta \alpha_{K K}\right)\left(\alpha_{\Gamma, L}-\alpha_{\Gamma, L}^{L}\right)}$.

$\sigma_{T}$ is the value of $\sigma$ for which $S=1$,

$$
\sigma_{T} \equiv \frac{\left(1-s-\beta_{K K}\right)}{\alpha_{K K}} .
$$

\subsubsection{Expressions for critical values of $\varepsilon_{\gamma}$}

$\varepsilon_{\gamma_{H}}$ is such that $D=1$, which is equivalent to:

$$
\varepsilon_{\gamma_{H}}=1+\frac{\left(1+\theta \alpha_{K, K}\right)\left(\sigma-\sigma_{H_{1}}\right)\left(\alpha_{\Gamma, L}^{H}-\alpha_{\Gamma, L}\right)}{\sigma\left(1+\theta \alpha_{K, K}\right)-\theta\left(1-s-\beta_{K, K}\right)} .
$$

$\varepsilon_{\gamma_{F}}$ is such that $1+T+D=0$. After some computations, we obtain:

$$
\varepsilon_{\gamma_{F}}=1+\frac{2\left(1+\theta \alpha_{K, K}\right)\left(\sigma_{F}-\sigma\right)\left(\alpha_{\Gamma, L}-\alpha_{\Gamma, L}^{L}\right)}{\sigma\left(2+\theta \alpha_{K, K}\right)-\theta\left(1-s-\beta_{K, K}\right)} .
$$

$\varepsilon_{\gamma_{T}}$ is such that $1-T+D=0$. After some computations, we obtain:

$$
\varepsilon_{\gamma_{T}}=1+\frac{\left(1-s-\beta_{K, K}\right)\left(\alpha_{\Gamma, L}^{*}-\alpha_{\Gamma, L}\right)}{\left(1-s-\beta_{K, K}-\sigma \alpha_{K, K}\right)} .
$$

\footnotetext{
${ }^{32}$ In Appendix 6.4, we show conditions for its existence and uniqueness.

${ }^{33}$ In Appendix 6.5 we we show conditions for its existence and uniqueness.
} 


\subsection{Existence of $\sigma_{H_{2}}$}

First recall that $\sigma_{H_{2}}$ is a value of $\sigma$ such that the half-line $\Delta$ line goes through point $C$, i.e., such that $\epsilon_{\gamma_{H}}=\epsilon_{\gamma_{T}}$ (see (21) and (23)). Further notice that if $\sigma_{H_{2}}$ exists it must be such that $\sigma_{H_{2}} \in\left(\sigma_{H_{1}},+\infty\right)$. Indeed, we can see geometrically that for $\sigma<\sigma_{H_{1}}$ the half line $\Delta$ starts above the line $(B C)$ and, since it points upwards to the right, if it crosses line $(A C)$ it does so above point $C$.

Consider first the case where $\alpha_{K, K}<0$. In this case, $S \in(0,1)$ for $\sigma \in\left(\frac{s-\beta_{L L}}{1+\alpha_{L L}},+\infty\right)$ and $\lim _{\sigma \rightarrow+\infty} S \in(0,1)$ (see Lemma 2). Hence, for $\sigma$ slightly higher that $\sigma_{H_{1}}$ the half line $\Delta$ crosses first $(B C)$ and then it crosses $(A C)$ above point $C$, i.e., $\epsilon_{\gamma_{H}}<\epsilon_{\gamma_{T}}$. Since $\lim _{\sigma \rightarrow+\infty} S \in(0,1)$, we can see geometrically that a solution $\sigma_{H_{2}} \in\left(\sigma_{H_{1}},+\infty\right)$ must exist and the number of these solutions is odd. Since the equation $\epsilon_{\gamma_{H}}=\epsilon_{\gamma_{T}}$ is a polynomial of degree 2, i.e. has at most two solutions, we deduce the uniqueness of $\sigma_{H_{2}}$, such that $\epsilon_{\gamma_{H}}<\epsilon_{\gamma_{T}}$ for $\sigma_{H_{1}}<\sigma<\sigma_{H_{2}}$, and $\epsilon_{\gamma_{H}}>\epsilon_{\gamma_{T}}$ for $\sigma>\sigma_{H_{2}}$.

Consider now that $\alpha_{K, K}=0$. In this case, we still have $S \in(0,1)$ for $\sigma \in\left(\frac{s-\beta_{L L}}{1+\alpha_{L L}},+\infty\right)$, although $\lim _{\sigma \rightarrow+\infty} S=1$ (see Lemma 2). Therefore, it is possible that the half line $\Delta$, for $\left(\sigma_{H_{1}},+\infty\right)$, always cross the line $(A C)$ above point $C$, and in this case $\sigma_{H_{2}}$ does not exist. Indeed, when $\alpha_{K, K}=0, \epsilon_{\gamma_{T}}$ does not depend on $\sigma$ (see (23)) and the equation $\epsilon_{\gamma_{H}}=\epsilon_{\gamma_{T}}$ is linear, having a solution $\sigma_{H_{2}}=\frac{s-\beta_{L L}}{1+\alpha_{L L}}+\frac{\left[\left(s-\beta_{L L}\right)-\theta\left(1+\alpha_{L L}\right)\left(1-s-\beta_{K K}\right)\right]\left(\alpha_{\Gamma L}^{*}-\alpha_{\Gamma L}^{* *}\right)}{\left(1+\alpha_{L L}\right)\left(\alpha_{\Gamma L}^{H}-\alpha_{\Gamma L}^{*}\right)}$. Hence, under Assumptions 1, 4 and 5.2, if $\alpha_{\Gamma L}^{H} \leq \alpha_{\Gamma L}^{*}$ there is no $\sigma_{H_{2}} \in\left(\frac{s-\beta_{L L}}{1+\alpha_{L L}},+\infty\right)$, i.e., the half line $\Delta$ cannot cross point $C$ and always go through $(A C)$ above point $C$. Therefore, we obtain $\epsilon_{\gamma_{H}}<\epsilon_{\gamma_{T}}$ for all $\sigma \in\left(\frac{s-\beta_{L L}}{1+\alpha_{L L}},+\infty\right)$. Otherwise, if $\alpha_{\Gamma L}^{H}>\alpha_{\Gamma L}^{*}$, there is a unique value $\sigma_{H_{2}} \in\left(\sigma_{H_{1}},+\infty\right)$ and we have $\epsilon_{\gamma_{H}}<\epsilon_{\gamma_{T}}$ for $\sigma<\sigma_{H_{2}}$, and $\epsilon_{\gamma_{H}}>\epsilon_{\gamma_{T}}$ for $\sigma>\sigma_{H_{2}}$.

Finally, consider that $\alpha_{K, K}>0$. Since under Assumption $7 \quad \sigma_{T}>\sigma_{H_{1}}$, we have $S \in(0,1)$ for $\sigma \in\left[\sigma_{H_{1}}, \sigma_{T}\right)$, and $S>1$ for $\sigma>\sigma_{T}$, with $\lim _{\sigma \rightarrow+\infty} S>1$ (see Lemma 2). We can see that for $\sigma$ slightly higher than $\sigma_{H_{1}}$ the half line $\Delta$ crosses $(B C)$ on the left of $C$ and $(A C)$ above point $C$, i.e., $\epsilon_{\gamma_{H}}<\epsilon_{\gamma_{T}}$. If there is a solution $\sigma_{H_{2}}$ to $\epsilon_{\gamma_{H}}=\epsilon_{\gamma_{T}}$ it has to satisfy $\sigma_{H_{2}} \in\left(\sigma_{H_{1}}, \sigma_{T}\right)$. Since $S>1$ for $\sigma>\sigma_{T}$, the half line $\Delta$ crosses $(B C)$ on the left of $C$, the existence of $\sigma_{H_{2}} \in\left(\sigma_{H_{1}}, \sigma_{T}\right)$ is not ensured and the number of solutions $\sigma_{H_{2}} \in\left(\sigma_{H_{1}}, \sigma_{T}\right)$ that satisfy $\epsilon_{\gamma_{H}}=\epsilon_{\gamma_{T}}$ must be even. The equation $\epsilon_{\gamma_{H}}=\epsilon_{\gamma_{T}}$ 
is equivalent to $g(\sigma)=0$, where:

$$
\begin{aligned}
g(\sigma) & \equiv \alpha_{K, K}\left(1+\theta \alpha_{K K}\right)\left(\alpha_{\Gamma, L-}^{H} \alpha_{\Gamma, L}\right)\left(\sigma-\sigma_{T}\right)\left(\sigma-\sigma_{H_{1}}\right) \\
& +\left[\sigma\left(1+\theta \alpha_{K, K}\right)-\theta\left(1-s-\beta_{K, K}\right)\right]\left(1-s-\beta_{K K}\right)\left(\alpha_{\Gamma L}^{*}-\alpha_{\Gamma L}\right)
\end{aligned}
$$

Under Assumptions 1 and 5, this function describes a convex parabola with $g\left(\sigma_{H_{1}}\right)>0, g\left(\sigma_{T}\right)>0$ and $g(+\infty)=+\infty$. Hence, either $g(\sigma)=0$ has two solutions (requiring $g^{\prime}\left(\sigma_{H_{1}}\right)<0$ ) or none (as it happens for instance when $\left.g^{\prime}\left(\sigma_{H_{1}} \geq 0\right)\right)$. As $g^{\prime}\left(\sigma_{H_{1}}\right) \geq 0$ is equivalent to:

$$
\sigma_{T}\left(\alpha_{\Gamma, L}^{H}-\alpha_{\Gamma, L}^{*}\right) \leq \sigma_{H_{1}}\left(\alpha_{\Gamma, L}^{H}-\alpha_{\Gamma, L}\right)
$$

when this inequality is satisfied, there is no solution to $g(\sigma)=0 .{ }^{34}$ This implies that for all $\sigma>\sigma_{H_{1}}$ the half-line $\Delta$ always goes above point $C$ and that, in particular, Assumption 8 is satisfied.

\subsection{Existence of $\sigma_{H_{3}}$}

Using (21) and (22), we have that $\epsilon_{\gamma_{H}} \geq \epsilon_{\gamma_{F}} \Leftrightarrow h(\sigma) \geq 0$, where:

$$
\begin{aligned}
h(\sigma) & \equiv\left[\sigma\left(2+\theta \alpha_{K, K}\right)-\theta\left(1-s-\beta_{K, K}\right)\right]\left(\alpha_{\Gamma, L}^{H}-\alpha_{\Gamma, L}\right)\left(\sigma-\sigma_{H_{1}}\right) \\
& +2\left[\sigma\left(1+\theta \alpha_{K, K}\right)-\theta\left(1-s-\beta_{K, K}\right)\right]\left(\alpha_{\Gamma, L}-\alpha_{\Gamma, L}^{L}\right)\left(\sigma-\sigma^{F}\right)
\end{aligned}
$$

By definition, $\sigma_{H_{3}}$ is a value of $\sigma$ such that $\epsilon_{\gamma_{H}}=\epsilon_{\gamma_{F}}$, therefore it must be a solution of $h(\sigma)=0$. Since $h(\sigma)$ is a polynomial of degree 2, the equation $h(\sigma)=0$ has at most two solutions. We limit our analysis to configuration (iii) since $\sigma_{H_{3}}$ is only relevant under this configuration. Since $\Delta$ is positively sloped pointing upwards, it can only go through point $B$ if its initial point in $\Delta_{1}$ is on the left of line $(A B)$, i.e., $\sigma_{H_{3}}<\sigma_{F}$. Also, the polynomial $h(\sigma)$ is a convex function of $\sigma$ since the coefficient of the quadratic term $\sigma^{2}$ is positive. ${ }^{35}$ We can see geometrically that if there is a $\sigma_{H_{3}}>\frac{s-\beta_{L L}}{1+\alpha_{L L}}$ then it must satisfy $\frac{s-\beta_{L L}}{1+\alpha_{L L}}<\sigma_{H_{1}}<\sigma_{H_{3}}<\sigma_{F}$. Using (25), and Assumptions 5, 6, we see that in this configuration $h\left(\sigma_{F}\right)>0$ and $h\left(\sigma_{H_{1}}\right)<0$. Therefore there is a unique $\sigma_{H_{3}} \in\left(\sigma_{H_{1}}, \sigma_{F}\right)$ such that $h\left(\sigma_{H_{3}}\right)=0$. By continuity, we have that $\epsilon_{\gamma_{H}}>\epsilon_{\gamma_{F}}$ for $\sigma_{F}>\sigma>\sigma_{H_{3}}$, and $\epsilon_{\gamma_{H}}<\epsilon_{\gamma_{F}}$ for $\sigma_{H_{1}}<\sigma<\sigma_{H_{3}}$.

\footnotetext{
${ }^{34}$ Note that, under Assumption 5.1, this inequality is always satisfied when $\alpha_{\Gamma, L}^{*}>\alpha_{\Gamma, L}^{H}$.

${ }^{35}$ Indeed, this coefficient is given by $c \equiv\left(2+\theta \alpha_{K K}\right)\left(\alpha_{\Gamma L}^{H}-\alpha_{\Gamma L}\right)+2\left(1+\theta \alpha_{K K}\right)\left(\alpha_{\Gamma L}-\right.$ $\left.\alpha_{\Gamma L}^{L}\right)$ which is positive under Assumptions 1, 5 and 6.
} 


\section{References}

[1] Alonso-Carrera, J., Caballé, J., and X. Raurich (2008), "Can Consumption Spillovers Be a Source of Equilibrium Indeterminacy?," Journal of Economic Dynamics and Control, 32, 2883-2902.

[2] d'Aspremont, C., Dos Santos Ferreira, R., and L.-A. Gérard-Varet (2000), "Endogenous Business Cycles and Business Formation with Strategic Investment," CORE DP 2000/53.

[3] Barinci, J.-P., and A. Chéron (2001), "Sunspot and the Business Cycle in a Finance Constrained Model," Journal of Economic Theory, 97, 3049.

[4] Basu, S. and J.G. Fernald (1997), " Returns to Scale in U.S. Production: Estimates and Implications," Journal of Political Economy, 105, 249-83.

[5] Benassy, J.-P. (1996), "Taste for Variety and Optimum Production Patterns in Monopolistic Competition, " Economics Letters 52, 41-47.

[6] Benhabib, J., and R. Farmer (1994), "Indeterminacy and Increasing Returns," Journal of Economic Theory, 63, 19-41.

[7] Benhabib, J., and R. Farmer (2000), "The Monetary Transmission Mechanism," Review of Economic Dynamics, 3, 523-550.

[8] Bils, M. (1987), "The Cyclical Behavior of Marginal Cost and Price," American Economic Review, 77, 838-855.

[9] Cazzavillan, G., T. Lloyd-Braga and P. Pintus (1998), "Multiple Steady States and Endogenous Fluctuations with Increasing Returns to Scale in Production," Journal of Economic Theory, 80, 60-107.

[10] Cazzavillan, G. (2001), "Indeterminacy and Endogenous Fluctuations with Arbitrarily Small Externalities," Journal of Economic Theory, 101, 133-157.

[11] Chari, V.V., P.J. Kehoe and E. R. McGrattan (2007), "Business Cycles Accounting," Econometrica, 75, 781-836.

[12] Coimbra, R., (1999), "Expectations-Driven Business Cycles Under the Efficiency Wages Hypothesis," unpublished Ph.D. dissertation, University of York. 
[13] Dos Santos Ferreira, R. and F. Dufourt (2006), "Free Entry and Business Cycles under the Influence of Animal Spirits," Journal of Monetary Economics, 53, 311-328.

[14] Dos Santos Ferreira, R. and T. Lloyd-Braga (2005), "Nonlinear Endogenous Fluctuations with Free Entry and Variable Markups," Journal of Economic Dynamics and Control, 29, 849-871.

[15] Dos Santos Ferreira, R. and T. Lloyd-Braga (2008), "Business Cycles with Free Entry Ruled by Animal Spirits," Journal of Economic Dynamics and Control, 32, 3502-3519.

[16] Dromel, N. and P. Pintus (2008), "Are Progressive Income Taxes Stabilizing?," Journal of Public Economic Theory, 10, 329-349.

[17] Drugeon, J.-P. and B. Wigniolle (1996), "Continuous-Time Sunspot Equilibria and Dynamics in a Model of Growth," Journal of Economic Theory, 69, 24-52.

[18] Duffy, J. and C. Papageorgiou (2000), "A Cross-country Empirical Investigation of the Aggregate Production Function Specification, " Journal of Economic Growth, 5, 87-120.

[19] Dufourt, F., Lloyd-Braga, T. and L. Modesto (2008), "Indeterminacy, Bifurcations and Unemployment Fluctuations, " Macroeconomic Dynamics, $12,75-89$.

[20] Dufourt, F., Lloyd-Braga, T. and L. Modesto (2007), "Sunspot Equilibria with Persistent Unemployment Fluctuations," mimeo.

[21] Gali, J. (1994), "Keeping Up with the Joneses: Consumption Externalities, Portfolio Choice and Asset Prices," Journal of Money Credit and Banking, 1-8.

[22] Gali, J. (1995), "Product Diversity, Endogenous Markups, and Development Traps," Journal of Monetary Economics, 36, 39-63.

[23] Gokan, Y. (2006), "Dynamic Effects of Government Expenditure in a Finance Constrained Economy," Journal of Economic Theory, 127, 323333.

[24] Grandmont, J.-M. (2008): "Negishi-Solow Efficiency Wages, Unemployment Insurance and Dynamic Deterministic Indeterminacy," International Journal of Economic Theory, 4, 247-272. 
[25] Grandmont, J.-M., P. Pintus and R. de Vilder (1998), "Capital-labour Substitution and Competitive Nonlinear Endogenous Business Cycles," Journal of Economic Theory, 80, 14-59.

[26] Guo, J.T. and K. Lansing (1998), "Indeterminacy and Stabilization Policy," Journal of Economic Theory, 82, 481-490.

[27] Hamermesh, D. S. (1996), Labor Demand, Princeton (N.J.) : Princeton University Press.

[28] Harrison, S. (2003), "Returns to Scale and Externalities in the Consumption and Investment Sectors", Review of Economic Dynamics, 6, 963-976.

[29] Jacobsen, H. J. (1998), "Endogenous Product Diversity and Endogenous Business Cycles, " Discussion Paper 98-15, University of Copenhagen.

[30] Kuhry, Y. (2001), "Endogenous Fluctuations in a Cournotian Monopolistic Competition Model with Free Entry and Market Power Variability, " Research in Economics, 55, 389-412.

[31] Ljungqvist, L. and H. Uhlig (2000), "Tax Policy and Aggregate Demand Management under Catching Up with the Joneses," American Economic Review, 90, 356-366.

[32] Lloyd-Braga, T. and L. Modesto (2007), "Indeterminacy in a Finance Constrained Unionized Economy," Journal of Mathematical Economics, $43,347-364$.

[33] Lloyd-Braga, T., L. Modesto and T. Seegmuller (2008), "Tax Rate Variability and Public Spending as Sources of Indeterminacy," Journal of Public Economic Theory, 10, 399-421.

[34] Martins, J.O. and S. Scarpetta (1999), "The Levels and Cyclical Behaviour of Mark-ups Across Countries and Market Structures," OECD Economics Department Working Papers 213.

[35] Maurer, J. and A. Meier (2008), "Smooth It Like the 'Joneses'? Estimating Peer-Group Effects in Intertemporal Consumption Choice," Economic Journal, 118, 454-476.

[36] Mendoza, E.G., A. Razin and L. Tesar (1994), "Effective Tax Rates in Macroeconomics: Cross-Country Estimates of Tax Rates on Factor Incomes and Consumption," Journal of Monetary Economics, 34, 297323. 
[37] Morrison, C.J. (1993),."Productive and Financial Performance in the U.S. Manufacturing Industries: An Integrated Structural Approach", Scandinavian Economic Journal, 60, 376-392.

[38] Nakajima, T. (2006), "Unemployment and Indeterminacy", Journal of Economic Theory, 126, 314-327.

[39] Pintus, P. (2003), "Aggregate Instability in the Fixed-Cost Approach to Public Spending," mimeo, Aix-Marseille.

[40] Portier, F. (1995) "Business Formation and Cyclical Markups in the French Business Cycle," Annales d'Economie et de Statistique, 37/38, $411 Đ 440$

[41] Rotemberg, J.J. and M. Woodford (1991),"Markups and the Business Cycle", in O. J. Blanchard and S. Fischer (eds), NBER Macroeconomics Annual, pp.63-128, Cambridge, Ma, MIT Press.

[42] Schmitt-Grohé, S. and M. Uribe (1997), "Balanced- Budget Rules, Distortionary Taxes, and Aggregate Instability," Journal of Political Economy, 105, 976-1000.

[43] Seegmuller, T. (2003), "Concurrence Imparfaite, Variabilité du Taux de Marge et Fluctuations Endogènes," Recherches Economiques de Louvain, 69, 371-386.

[44] Seegmuller, T. (2008), "Taste for Variety and Endogenous Fluctuations in a Monopolistic Competition Model," Macroeconomic Dynamics, 12, 561-577.

[45] Seegmuller, T. (2009), "Capital-labour Substitution and Endogenous Fluctuations: a Monopolistic Competition Approach with Variable Markup, " Japanese Economic Review 60, 301-319.

[46] Volkerink, B., J-E Sturm and J. de Haan (2002), "Tax Ratios in Macroeconomics: Do Taxes Really Matter?", Empirica, 29, 209-224.

[47] Wang, P. and Y. Wen (2008), "Imperfect Competition and Indeterminacy of Aggregate Output," Journal of Economic Theory, 143, 519-540.

[48] Weder, M. (2000a), "Animal Spirits, Technology Shocks and the Business Cycle," Journal of Economic Dynamics and Control, 24, 273-295.

[49] Weder, M. (2000b), "Consumption Externalities, Production Externalities and Indeterminacy, " Metroeconomica, 51, 435-453. 
[50] Weder, M. (2004), "A Note on Conspicuous Leisure, Animal Spirits and Endogenous Cycles, " Portuguese Economic Journal, 3, 1-13.

[51] Woodford, M., (1986), "Stationary Sunspot Equilibria in a Finance Constrained Economy," Journal of Economic Theory, 40, 128-137.

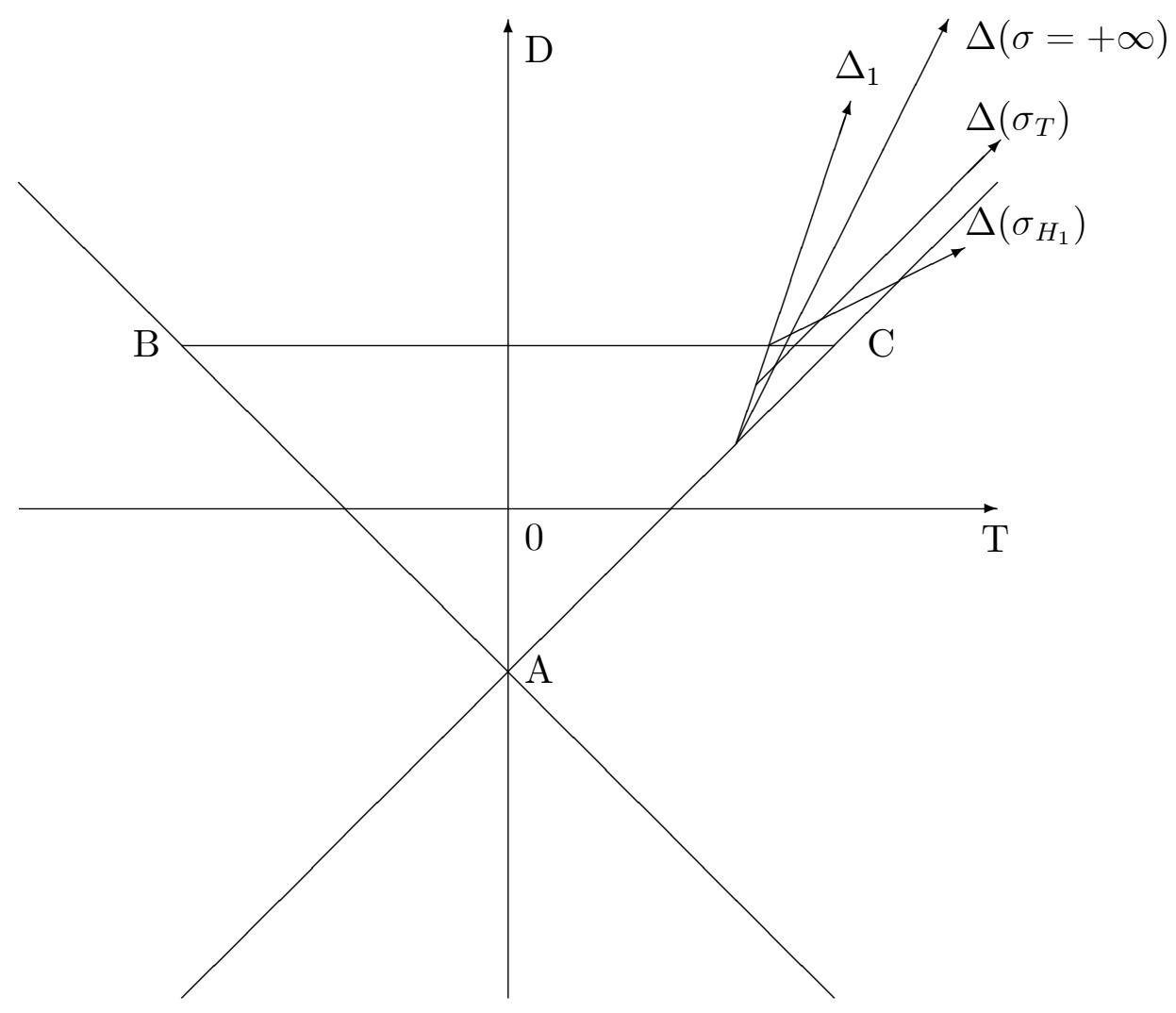

Figure 1: Configuration $(i)$, with $\alpha_{K, K}>0$ 


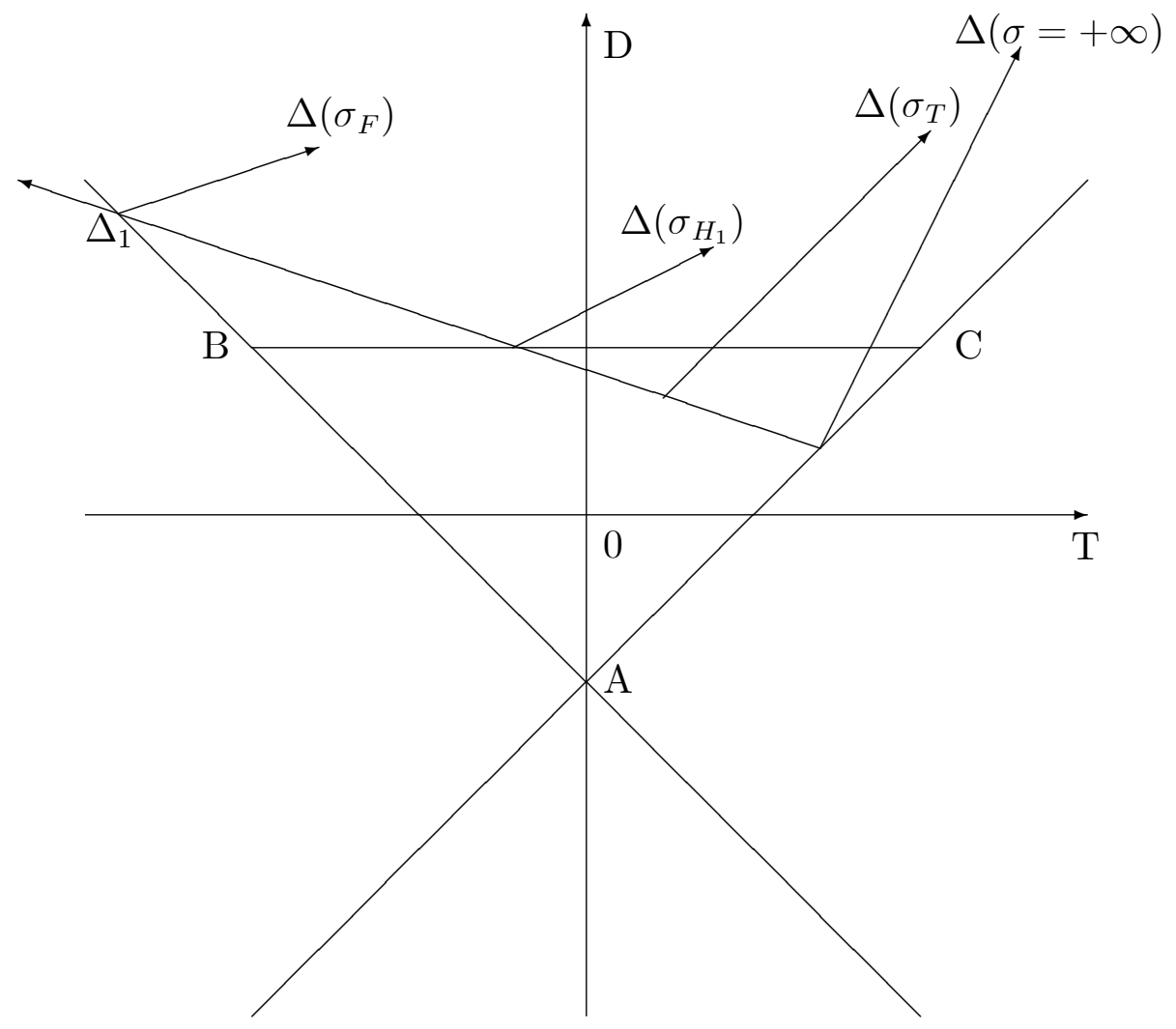

Figure 2: Configuration (ii), with $\alpha_{K, K}>0$ 


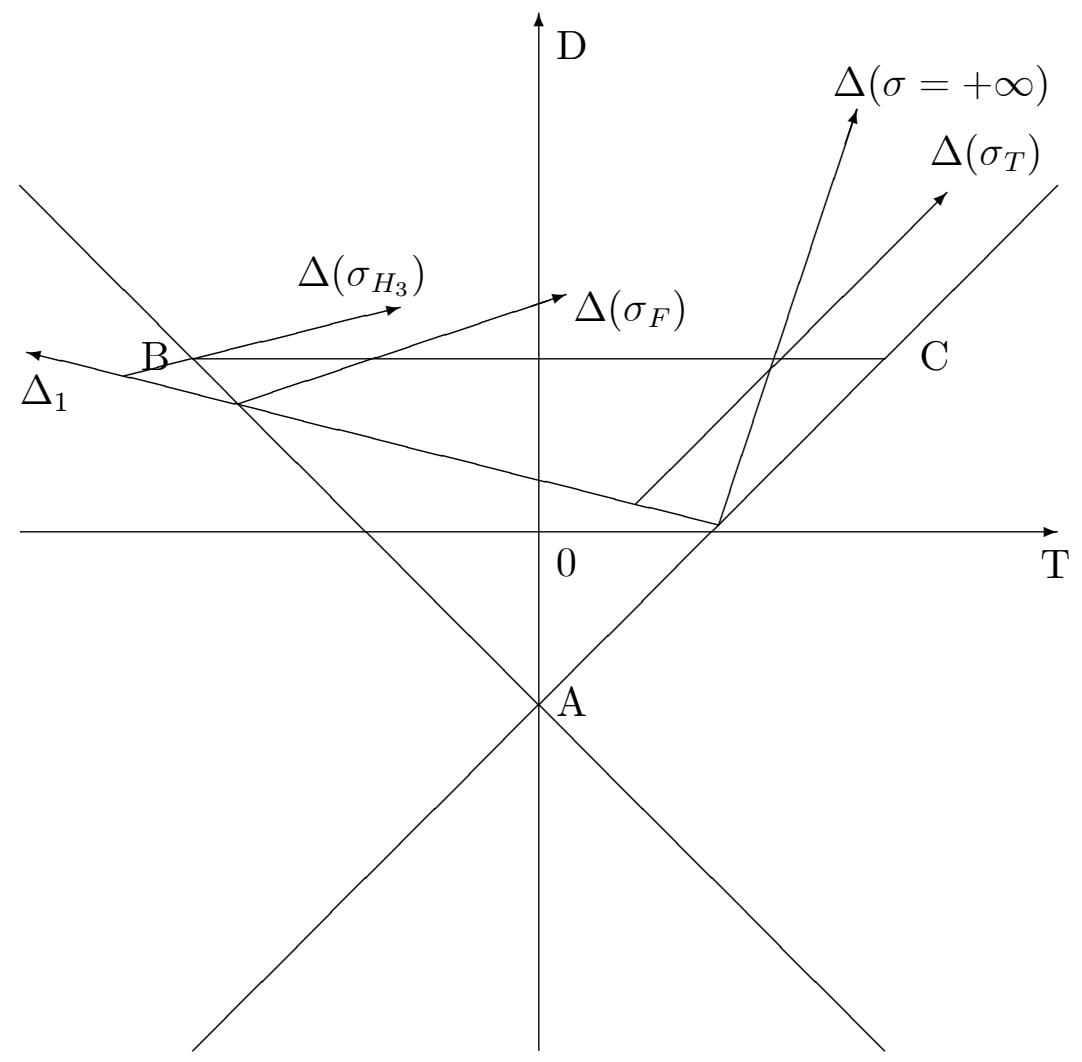

Figure 3: Configuration (iii), with $\alpha_{K, K}>0$ 